\title{
Boundary regularity for degenerate and singular parabolic equations
}

\author{
Anders Björn \\ Department of Mathematics, Linköpings universitet, \\ SE-58183 Linköping, Sweden; anders.bjorn@liu.se \\ Jana Björn \\ Department of Mathematics, Linköpings universitet, \\ SE-58183 Linköping, Sweden; jana.bjorn@liu.se \\ Ugo Gianazza \\ Department of Mathematics "F. Casorati", Università di Pavia, \\ via Ferrata 1, IT-27100 Pavia, Italy; gianazza@imati.cnr.it \\ Mikko Parviainen \\ Department of Mathematics and Statistics, University of Jyväskylä, \\ P.O. Box 35 (MaD), FI-40014 Jyväskylä, Finland; mikko.j.parviainen@jyu.fi
}

\begin{abstract}
We characterise regular boundary points of the parabolic $p$-Laplacian in terms of a family of barriers, both when $p>2$ and $1<p<2$. Due to the fact that $p \neq 2$, it turns out that one can multiply the $p$-Laplace operator by a positive constant, without affecting the regularity of a boundary point. By constructing suitable families of barriers, we give some simple geometric conditions that ensure the regularity of boundary points.
\end{abstract}

Key words and phrases: barrier family, cone condition, degenerate parabolic, exterior ball condition, $p$-Laplacian, Perron's method, Petrovskil criterion, regular boundary point, singular parabolic.

2010 Mathematics Subject Classification: Primary: 35K20; Secondary: 31B25, 35B65, 35K65, 35K67, 35K92.

\section{Introduction}

A boundary point is regular with respect to a partial differential equation if all solutions to the Dirichlet problem attain their continuous boundary values continuously at that point. The characterisation of regular boundary points for different partial differential equations has a very long history. Wiener gave a necessary and sufficient condition, the Wiener criterion, for the boundary regularity in the context of the Laplace equation in his celebrated 1924 paper [42]. Evans and Gariepy [13] settled the question about the boundary regularity for the heat equation, but the boundary regularity for $p$-parabolic type equations in terms of an explicit Wiener type criterion is a long standing open problem. In this paper, we characterise regular boundary points for the $p$-parabolic equation, $1<p<\infty$,

$$
\frac{\partial u}{\partial t}=\operatorname{div}\left(|\nabla u|^{p-2} \nabla u\right)
$$


in terms of a family of barrier functions (Theorem 3.3).

The parabolic boundary regularity is a quite delicate question, and this is already apparent, when dealing with the one-dimensional linear heat equation. Indeed, a boundary point can be regular for the equation

$$
\frac{\partial u}{\partial t}=\frac{\partial^{2} u}{\partial x^{2}}
$$

but irregular for its (still linear) cousin

$$
2 \frac{\partial u}{\partial t}=\frac{\partial^{2} u}{\partial x^{2}}
$$

This can be seen using Petrovskiı̌'s criterion for the one-dimensional heat equation, see $[40,41]$. The interested reader can refer to the historical introduction presented by Galaktionov [15], in particular for the improvements with respect to the original result of [40], that Petrovskil proved in [41].

Such a behaviour might raise a serious doubt on all the attempts for proving the regularity of boundary points for parabolic problems, let alone a parabolic Wiener's criterion, based on estimates with unspecified constants. However, we show in Theorem 3.6, perhaps surprisingly, that in the nonlinear case, the regularity of a boundary point is not affected by multiplicative constants on one side of the equation. We also derive several concrete characterisations of the regularity of boundary points: by constructing an explicit family of barriers, we show that the exterior ball condition and several exterior cone conditions imply the regularity of a boundary point. Moreover, the barrier family characterisation implies that the regularity of a boundary point is of local nature and that the future does not affect the regularity of a point. Finally, we establish a Petrovskiı type regularity condition for the latest boundary point.

Perron's method [39], also called the Perron-Wiener-Brelot method, originally developed for the Laplace equation, has become a fundamental tool in the study of Dirichlet boundary value problems for various elliptic and parabolic partial differential equations. The idea is to construct an upper solution of the Dirichlet problem as an infimum of a certain upper class of supersolutions. A lower solution is constructed similarly using a lower class of subsolutions, and when the upper and lower solutions coincide we obtain a reasonable solution. In the Perron method, the boundary regularity is essentially a separate problem from the existence of a solution.

Systematic use of barrier functions as a tool for studying boundary regularity seems to date back to the 1912 work of Lebesgue [29]. Later, Lebesgue [30] characterised regular boundary points in terms of barriers for the linear Laplace equation. In the elliptic setting, the extension of Perron's method and the method of barriers to the nonlinear $p$-Laplacian was initiated by Granlund, Lindqvist and Martio in [17] and developed in a series of papers (see, for example, the accounts given in Heinonen-Kilpeläinen-Martio [18] and Björn-Björn [4]).

Coming to the heat equation, in much of the existing literature, the subject has been addressed as an analog to the classical theory for the Laplace equation (see e.g. Doob [11]) or as an example of abstract potential theory (see e.g. BliedtnerHansen [8]). However, the recent book by Watson [43] deals with heat potential theory as a subject on its own.

The potential theory for $p$-parabolic type equations was initiated by Kilpeläinen and Lindqvist in [21]. They established the parabolic Perron method, and also suggest a boundary regularity condition in terms of one barrier function. Even if the single barrier criterion has turned out to be problematic, ${ }^{1}$ our paper owes a lot of inspiration and techniques to [21], as well as [32].

\footnotetext{
${ }^{1}$ We thank P. Lindqvist for bringing this problem to our attention.
} 
The paper is organised as follows: Section 2 is devoted to some preliminary material. In particular, we recall the different concepts of solutions - weak supersolutions and superparabolic functions - as well as the Perron method. Section 3 deals with the boundary regularity, the definition of a family of barriers and the characterisation of a regular boundary point in terms of barriers, with some related properties.

The following sections are devoted to simple geometric criteria for the regularity of boundary points, which are all derived by constructing suitable families of barriers. In particular, the exterior ball condition of Section 4 states that if there is an exterior ball touching the domain at a point, then this point is a regular boundary point with two exceptions: Consider the Dirichlet problem in a space time cylinder $\Omega \times(0, T)$. The boundary values are given on the lateral boundary $\partial \Omega \times(0, T)$ as well as on the initial boundary $\Omega \times\{0\}$. However, the solution itself determines the values on $\Omega \times\{T\}$. Thus, evidently, the point of contact should not be the south pole of the exterior ball. However, curiously also the 'north pole' as a point of contact causes difficulties when using the natural family of barriers. By different means, one easily sees that for example $(x, t) \in \Omega \times\{0\}$ is a regular point in the cylindrical case. However, if the initial boundary is for example a half sphere (like at least roughly in a soda can), then it would be interesting to know if the north pole of the initial boundary is regular.

Section 5 deals with cone conditions, while Section 6 deals with a Petrovskiu type condition for $p>2$. Petrovskiu [41] showed that $(0,0)$ is regular for the heat equation with respect to

$$
\{(x, t):|x|<2(1+\varepsilon) \sqrt{-t} \sqrt{\log |\log (-t)|} \text { and }-1 \leq t<0\},
$$

if $\varepsilon=0$, while it is irregular if $\varepsilon>0$. In Section 6 we obtain a similar result for $p>2$. We have not been able to obtain a Petrovskiu type condition for $1<p<2$, but in Section 7 we deduce a somewhat weaker result. We end the paper by giving a list of some open problems in Section 8.

Acknowledgement. A. B. and J. B. are supported by the Swedish Research Council, and M. P. by the Academy of Finland. Part of this research was done during several visits: of M. P. to Linköpings universitet in 2007, of A. B. to Università di Pavia in 2011, of U. G. to University of Jyväskylä in 2012, and while all authors visited Institut Mittag-Leffler in 2013.

\section{Preliminaries}

Let $\Theta$ be a bounded nonempty open set in $\mathbf{R}^{n+1}, 1<p<\infty$, and $z=(x, t) \in \mathbf{R}^{n+1}$. We consider the equation

$$
\frac{\partial u}{\partial t}=\Delta_{p} u:=\operatorname{div}\left(|\nabla u|^{p-2} \nabla u\right)
$$

where the gradient $\nabla u$ and the $p$-Laplacian $\Delta_{p}$ are taken with respect to $x$. This equation is degenerate if $p>2$ and singular if $1<p<2$. For $p=2$ it is the usual heat equation.

Observe that if $u$ satisfies (2.1), and $a \in \mathbf{R}$, then $-u$ and $u+a$ also satisfy (2.1), but (in general) au does not.

In what follows, unless otherwise stated, $Q$ stands for a box $Q=\left(a_{1}, b_{1}\right) \times \ldots \times$ $\left(a_{n}, b_{n}\right)$ in $\mathbf{R}^{n}$, and the sets $Q_{T}=Q \times(0, T)$ and $Q_{t_{1}, t_{2}}=Q \times\left(t_{1}, t_{2}\right)$ are called space-time boxes. Further, $B\left(\xi_{0}, r\right)=\left\{z \in \mathbf{R}^{n+1}:\left|z-\xi_{0}\right|<r\right\}$ stands for the usual Euclidean ball in $\mathbf{R}^{n+1}$. 
Let $U$ be an open set in $\mathbf{R}^{n}$. The parabolic boundary of a cylinder $U_{t_{1}, t_{2}}:=$ $U \times\left(t_{1}, t_{2}\right) \subset \mathbf{R}^{n+1}$ is

$$
\partial_{p} U_{t_{1}, t_{2}}=\left(\bar{U} \times\left\{t_{1}\right\}\right) \cup\left(\partial U \times\left(t_{1}, t_{2}\right]\right) .
$$

We define the parabolic boundary of a finite union of open cylinders $U_{t_{1}^{i}, t_{2}^{i}}^{i}$ as follows

$$
\partial_{p}\left(\bigcup_{i} U_{t_{1}^{i}, t_{2}^{i}}^{i}\right):=\left(\bigcup_{i} \partial_{p} U_{t_{1}^{i}, t_{2}^{i}}^{i}\right) \backslash \bigcup_{i} U_{t_{1}^{i}, t_{2}^{i}}^{i}
$$

If $D^{\prime}$ is a bounded open subset of $D$ and the closure of $D^{\prime}$ belongs to $D$, we write $D^{\prime} \Subset D$. Note that the parabolic boundary is by definition compact.

Let $U$ be a bounded open set in $\mathbf{R}^{n}$. As usual, $W^{1, p}(U)$ denotes the space of real-valued functions $f$ such that $f \in L^{p}(U)$ and the distributional first partial derivatives $\partial f / \partial x_{i}, i=1,2, \ldots, n$, exist in $U$ and belong to $L^{p}(U)$. We use the norm

$$
\|f\|_{W^{1, p}(U)}=\left(\int_{U}|f|^{p} d x+\int_{U}|\nabla f|^{p} d x\right)^{1 / p}
$$

The Sobolev space with zero boundary values, $W_{0}^{1, p}(U)$, is the closure of $C_{0}^{\infty}(U)$ with respect to the Sobolev norm.

By the parabolic Sobolev space $L^{p}\left(t_{1}, t_{2} ; W^{1, p}(U)\right)$, with $t_{1}<t_{2}$, we mean the space of functions $u(x, t)$ such that the mapping $x \mapsto u(x, t)$ belongs to $W^{1, p}(U)$ for almost every $t_{1}<t<t_{2}$ and the norm

$$
\left(\int_{t_{1}}^{t_{2}} \int_{U}|u(x, t)|^{p}+|\nabla u(x, t)|^{p} d x d t\right)^{1 / p}
$$

is finite. The definition of the space $L^{p}\left(t_{1}, t_{2} ; W_{0}^{1, p}(U)\right)$ is similar. Analogously by the space $C\left(t_{1}, t_{2} ; L^{p}(U)\right)$, with $t_{1}<t_{2}$, we mean the space of functions $u(x, t)$, such that the mapping $t \mapsto \int_{U}|u(x, t)|^{p} d x$ is continuous in the time interval $\left[t_{1}, t_{2}\right]$. (The gradient $\nabla$ and divergence div are always taken with respect to the $x$-variables in this paper.) We can now introduce the notion of weak solution.

Definition 2.1. A function $u: \Theta \rightarrow[-\infty, \infty]$ is a weak solution to equation (2.1) if whenever $U_{t_{1}, t_{2}} \Subset \Theta$ is an open cylinder, we have $u \in C\left(t_{1}, t_{2} ; L^{2}(U)\right) \cap$ $L^{p}\left(t_{1}, t_{2} ; W^{1, p}(U)\right)$, and $u$ satisfies the integral equality

$$
\int_{t_{1}}^{t_{2}} \int_{U}|\nabla u|^{p-2} \nabla u \cdot \nabla \varphi d x d t-\int_{t_{1}}^{t_{2}} \int_{U} u \frac{\partial \varphi}{\partial t} d x d t=0 \quad \text { for all } \varphi \in C_{0}^{\infty}\left(U_{t_{1}, t_{2}}\right) .
$$

Continuous weak solutions are called p-parabolic functions.

A function $u$ is a weak supersolution (subsolution), if whenever $U_{t_{1}, t_{2}} \Subset \Theta$ we have $u \in L^{p}\left(t_{1}, t_{2} ; W^{1, p}(U)\right)$, and the left-hand side above is nonnegative (nonpositive) for all nonnegative $\varphi \in C_{0}^{\infty}\left(U_{t_{1}, t_{2}}\right)$.

In the following, for simplicity, we will often omit weak, when talking of weak (super)solutions.

Locally bounded solutions are locally Hölder continuous (see DiBenedetto [9], Chapters III and IV). For $p>\frac{2 n}{n+2}$ the notion of solution automatically yields local boundedness, whereas for $1<p \leq \frac{2 n}{n+2}$ explicit unbounded solutions are known, and in order to guarantee boundedness, an extra assumption on $u$ is needed (see the discussions in [9], Chapter V, and DiBenedetto-Gianazza-Vespri [10], Appendix A). Although it plays no role in the following, it is worth mentioning that nonnegative solutions satisfy proper forms of Harnack inequalities (see [10], and also Kuusi [27]), 
and locally bounded gradients of solutions are locally Hölder continuous (see [9], Chapter IX).

The so-called Barenblatt solution [2] to equation (2.1)

$\mathcal{B}_{p}(x, t)=t^{-n / \lambda}\left(C-\frac{p-2}{p} \lambda^{1 /(1-p)}\left(\frac{|x|}{t^{1 / \lambda}}\right)^{p /(p-1)}\right)_{+}^{(p-1) /(p-2)}, \quad \lambda=n(p-2)+p$,

is used in this paper. Even though it was introduced in the context of degenerate equations for $p>2$, it is well defined also for $p<2$, provided that $\lambda>0$, which requires $\frac{2 n}{n+1}<p<2$.

Definition 2.2. A function $u: \Theta \rightarrow(-\infty, \infty]$ is $p$-superparabolic if

(i) $u$ is lower semicontinuous;

(ii) $u$ is finite in a dense subset of $\Theta$;

(iii) $u$ satisfies the following comparison principle on each space-time box $Q_{t_{1}, t_{2}} \Subset$ $\Theta$ : If $h$ is $p$-parabolic in $Q_{t_{1}, t_{2}}$ and continuous on $\bar{Q}_{t_{1}, t_{2}}$, and if $h \leq u$ on $\partial_{p} Q_{t_{1}, t_{2}}$, then $h \leq u$ in the whole $Q_{t_{1}, t_{2}}$.

A $p$-subparabolic function $v: \Theta \rightarrow[-\infty, \infty)$ is defined analogously, except that $v$ is upper semicontinuous and the inequalities are reversed, i.e. we require that if $h \geq v$ on $\partial_{p} Q_{t_{1}, t_{2}}$, then $h \geq v$ in the whole $Q_{t_{1}, t_{2}}$. Equivalently, $v$ is $p$-subparabolic if $-v$ is $p$-superparabolic.

It is also worth mentioning that the definition of $p$-superparabolic functions given here is equivalent to the modern definition of viscosity supersolutions as shown in Juutinen-Lindqvist-Manfredi [20].

Theorem 2.3. Consider a parabolic cylinder $G_{T}=G \times(0, T)$, where $G$ is a bounded domain in $\mathbf{R}^{n}$ with a Lipschitz boundary $\partial G$, and let $h \in C\left(\partial_{p} G_{T}\right)$. Then there is a unique p-parabolic function $u \in C\left(\bar{G}_{T}\right)$ that is continuous in $G_{T}$ and takes the boundary values $u=h$ on the parabolic boundary $\partial_{p} G_{T}$. Moreover, if $h$ belongs to $C\left(t_{1}, t_{2} ; L^{2}(G)\right) \cap L^{p}\left(t_{1}, t_{2} ; W^{1, p}(G)\right)$, then so does $u$.

We will need this result explicitly when proving the parabolic comparison principle in Theorem 2.4, but we also rely on it implicitly since it is used (at least for boxes) when obtaining some results we quote from, e.g., Kilpeläinen-Lindqvist [21].

Although it is well-known in the literature, to our knowledge a full proof which covers the whole range $1<p<\infty$ is given only in Ivert [19], Theorem 3.2, which in turn relies on Fontes [14].

In the proof of the 'pasting lemma' (Lemma 2.9), we need the parabolic comparison principle which is essentially stated in [21], Lemma 4.3, and [25], Theorem 4.8. However, as a full proof seems to be difficult to find in the literature, we have chosen to write it down in details. In particular, there is a subtle technical issue when proceeding in time: At a first approach, one might be tempted to consider the Euclidean boundary of the whole finite union of space-time boxes at once, construct a suitable $p$-parabolic comparison function $h$, and then compare $v$ as well as $u$ with $h$. However, such a comparison function, continuous on the whole Euclidean closure does not necessarily exist. Consider for example

$$
\Xi=((0,3) \times(0,1)) \cup((1,2) \times(0,2)) \subset \mathbf{R}^{1+1},
$$

with the boundary data $\psi=0$ on $\partial_{p}((0,3) \times(0,1))$ and $\psi=1$ on $\{1,2\} \times[1,2]$. The function $\psi$ is continuous on $\partial_{p} \Xi$ (and easily extends to a continuous function on the whole of $\partial \Xi$ ) but any $p$-parabolic function in $(0,3) \times(0,1)$ with $\psi$ as boundary values must be identically zero in $(0,3) \times(0,1)$, and thus cannot continuously attain the boundary value 1 at the corner points $(1,1)$ and $(2,1)$. 
Theorem 2.4. (Parabolic comparison principle) Let $\Theta$ be an open bounded set in $\mathbf{R}^{n+1}$. Suppose that $u$ is $p$-superparabolic and $v$ is $p$-subparabolic in $\Theta$. Let $T \in \mathbf{R}$ and assume that

$$
\infty \neq \limsup _{\Theta \ni(y, s) \rightarrow(x, t)} v(y, s) \leq \liminf _{\Theta \ni(y, s) \rightarrow(x, t)} u(y, s) \neq-\infty
$$

for all $(x, t) \in\{(x, t) \in \partial \Theta: t<T\}$. Then $v \leq u$ in $\{(x, t) \in \Theta: t<T\}$.

For the proof we will rely on the following lemma.

Lemma 2.5. Let $U=\bigcup_{i} Q^{i}$ be a finite union of boxes and let $U_{T}:=U \times(0, T)$ be the corresponding cylinder. Also let $u$ be a $p$-superparabolic function in a neighbourhood of $\bar{U}_{T}$, and $h \in C\left(\bar{U}_{T}\right)$ be a function which is p-parabolic in $U_{T}$ and such that $h \leq u$ on $\partial_{p} U_{T}$. Then $h \leq u$ in $U_{T}$.

This lemma was stated and proved for the supercritical case $p>\frac{2 n}{n+2}$ in KorteKuusi-Parviainen [25], Lemma 4.1. A careful check of the proof reveals that the requirement $p>\frac{2 n}{n+2}$ is used to ensure that $v-u \in L^{2}$ in the proof of the comparison principle between sub- and supersolutions in Lemma 3.5 in [25], which the proof of Lemma 4.1 relies on through the proof of Lemma 3.6 in [25]. To also cover the range $1<p \leq \frac{2 n}{n+2}$ one can proceed as follows: First assume that $u$ is bounded (that $h$ above is bounded is automatic). Then the $L^{2}$ integrability is immediate for $u$ and $h$. The proof of Lemma 4.1 in [25] is also relying on an existence result for obstacle problems, which is Theorem 2.8 therein and whose proof can be found in KorteKuusi-Siljander [26, Theorem 3.1] or in Lindqvist-Parviainen [35, Theorem 3.2]. These existence theorems in turn rely on Lemma 3.5 in [25] (but only applied with bounded functions so that the $L^{2}$ integrability is automatic) and on a convergence result for supersolutions (Theorem 5.3 in [25]), whose proof also applies in the subcritical case. Thus, we have obtained Lemma 2.5 above with $u$ bounded for all $1<p<\infty$.

Finally, to cover also the unbounded case when $p \leq \frac{2 n}{n+2}$, let $m=\sup _{\bar{U}_{T}} h$ and $v=\min \{u, m\}$. That $v$ is $p$-superparabolic is immediate from the definition. We can then apply Lemma 2.5 with the bounded functions $v$ and $h$, which yields that $h \leq v$ in $U_{T}$. Since $v \leq u$, this concludes the proof also for the unbounded case.

Proof of Theorem 2.4. Let $\varepsilon>0$ and

$$
E=\{(x, t) \in \Theta: t \leq T-\varepsilon \text { and } v(x, t)>u(x, t)+\varepsilon\} .
$$

By (2.2), together with the compactness of $\{(x, t) \in \partial \Theta: t \leq T-\varepsilon\}$, we conclude that $\bar{E}$ is a compact subset of $\Theta$. Assume that $E \neq \varnothing$, and let

$$
T_{0}=\inf \{t:(x, t) \in E\}=\min \{t:(x, t) \in \bar{E}\} .
$$

Since $\bar{E}$ is compact, we can find finitely many space-time boxes $Q_{t_{1}^{i}, t_{2}^{i}}^{i} \Subset \Theta$ such that $\bigcup_{i=1}^{N} Q_{t_{1}^{i}, t_{2}^{i}}^{i} \supset \bar{E}$, where $t_{1}^{i} \neq T_{0}$ and $T_{0}<t_{2}^{i}<T, i=1,2, \ldots, N$. By changing the cover, we may assume that $S:=\left\{t_{j}^{i}: j=1,2\right.$ and $\left.i=1,2, \ldots, N\right\}$ only contains one value, say $\sigma$, less than $T_{0}$. Let $m$ be the number of boxes $Q_{t_{1}^{i}, t_{2}^{i}}^{i}$ with $t_{1}^{i}=\sigma$, and assume that these are ordered first. Let $\Xi:=\bigcup_{i=1}^{m} Q_{\sigma, \tau}^{i} \supset\{(x, t) \in \bar{E}: t<\tau\}$, where $\tau=\min \left\{t \in S: t>T_{0}\right\}>T_{0}$.

In particular, the parabolic boundary $\partial_{p} \Xi \subset \Theta \backslash E$, and hence $v \leq u+\varepsilon$ on $\partial_{p} \Xi$. Thus there exists a continuous function $\psi$ on $\partial_{p} \Xi$ such that $v \leq \psi \leq u+\varepsilon$. By Theorem 2.3, we can find a function $h \in C(\bar{\Xi})$ which is $p$-parabolic in $\Xi$ and continuously attains its boundary values $h=\psi$ on $\partial_{p} \Xi$. Lemma 2.5 applied in $\Xi$ to $u+\varepsilon$ and $h$, and to $-v$ and $-h$, shows that $v \leq h \leq u+\varepsilon$ in $\Xi$.

Thus $\Xi \cap E=\varnothing$, and so $T_{0} \geq \tau$, a contradiction. Hence $E$ must be empty, and letting $\varepsilon \rightarrow 0$ concludes the proof. 
A direct consequence of Theorem 2.4 is the following comparison principle, which can be considered as a sort of elliptic version of the comparison principle, since it does not acknowledge the presence of the parabolic boundary. This elliptic comparison principle is in fact equivalent to the fundamental inequality $\underline{H} f \leq \bar{H} f$ between lower and upper Perron solutions (see Definition 2.8 below).

Theorem 2.6. (Elliptic-type comparison principle) Suppose that $u$ is p-superparabolic and $v$ is $p$-subparabolic in $\Theta$. If

$$
\infty \neq \limsup _{\Theta \ni(y, s) \rightarrow(x, t)} v(y, s) \leq \liminf _{\Theta \ni(y, s) \rightarrow(x, t)} u(y, s) \neq-\infty
$$

for all $(x, t) \in \partial \Theta$, then $v \leq u$ in $\Theta$.

The connection between $p$-superparabolic functions and weak supersolutions is a delicate issue, see for example Kinnunen-Lindqvist [23], [24], Kuusi [28] and the survey in Lindqvist [33]. However, to conclude that our continuous barriers below are $p$-superparabolic for all $p>1$, we only need to check that they are weak supersolutions and then use the following comparison principle for weak (sub/super)solutions, see Lemma 3.1 of Kilpeläinen-Lindqvist [21] and Lemma 3.5 of Korte-Kuusi-Parviainen [25].

Lemma 2.7. Suppose that $u$ is a weak supersolution and $v$ is a weak subsolution to (2.1) in a space-time cylinder $U_{t_{1}, t_{2}}$, where $U \subset \mathbf{R}^{n}$ is an open set. If $u$ and $-v$ are lower semicontinuous on $\bar{U}_{t_{1}, t_{2}}$ and $v \leq u$ on the parabolic boundary $\partial_{p} U_{t_{1}, t_{2}}$, then $v \leq u$ a.e. in $U_{t_{1}, t_{2}}$.

Let us now come to Perron's method for (2.1). For us it will be enough to consider Perron solutions for bounded functions, so for simplicity we restrict ourselves to this case throughout this paper.

Definition 2.8. Given a bounded function $f: \partial \Theta \rightarrow \mathbf{R}$, let the upper class $\mathcal{U}_{f}$ be the set of all $p$-superparabolic functions $u$ on $\Theta$ which are bounded below and such that

$$
\liminf _{\Theta \ni \eta \rightarrow \xi} u(\eta) \geq f(\xi) \quad \text { for all } \xi \in \partial \Theta .
$$

Define the upper Perron solution of $f$ by

$$
\bar{H} f(\xi)=\inf _{u \in \mathcal{U}_{f}} u(\xi), \quad \xi \in \Theta .
$$

Similarly, let the lower class $\mathcal{L}_{f}$ be the set of all $p$-subparabolic functions $u$ on $\Theta$ which are bounded above and such that

$$
\limsup _{\Theta \ni \eta \rightarrow \xi} u(\eta) \leq f(\xi) \text { for all } \xi \in \partial \Theta,
$$

and define the lower Perron solution of $f$ by

$$
\underline{H} f(\xi)=\sup _{u \in \mathcal{L}_{f}} u(\xi), \quad \xi \in \Theta .
$$

Note that we have an elliptic-type boundary condition on the full boundary, not just a condition on the possibly smaller parabolic boundary, whenever it is defined.

It follows from the elliptic-type comparison principle in Theorem 2.6 that $v \leq$ $u$ whenever $u \in \mathcal{U}_{f}$ and $v \in \mathcal{L}_{f}$. Hence $\underline{H} f \leq \bar{H} f$. Moreover, KilpeläinenLindqvist [21], Theorem 5.1, proved that both $\underline{H} f$ and $\bar{H} f$ are $p$-parabolic.

The following lemma is useful when constructing new $p$-superparabolic functions. 
Lemma 2.9. (Pasting lemma) Let $G \subset \Theta$ be open. Also let $u$ and $v$ be $p$-superparabolic in $\Theta$ and $G$, respectively, and let

$$
w= \begin{cases}\min \{u, v\} & \text { in } G, \\ u & \text { in } \Theta \backslash G .\end{cases}
$$

If $w$ is lower semicontinuous, then $w$ is p-superparabolic in $\Theta$.

Proof. Since $-\infty<w \leq u, w$ is finite in a dense subset of $\Theta$, and we only have to obtain the comparison principle. Therefore, let $Q_{t_{1}, t_{2}} \Subset \Theta$ be a space-time box, and $h \in C\left(\bar{Q}_{t_{1}, t_{2}}\right)$ be $p$-parabolic in $Q_{t_{1}, t_{2}}$ and such that $h \leq w$ on $\partial_{p} Q_{t_{1}, t_{2}}$. Since $h \leq u$ on $\partial_{p} Q_{t_{1}, t_{2}}$ and $u$ is $p$-superparabolic, we directly have that $h \leq u$ in $Q_{t_{1}, t_{2}}$.

Next let $\widetilde{G}=Q_{t_{1}, t_{2}} \cap G$ and $(x, t) \in\left\{(x, t) \in \partial \widetilde{G}: t<t_{2}\right\}$. If $(x, t) \in G$, then $(x, t) \in \partial_{p} Q_{t_{1}, t_{2}}$ and thus by the lower semicontinuity of $v$,

$$
\liminf _{\widetilde{G} \ni(y, s) \rightarrow(x, t)} v(y, s) \geq v(x, t) \geq h(x, t) .
$$

On the other hand, if $(x, t) \notin G$, then, by the lower semicontinuity of $w$,

$$
\liminf _{\widetilde{G} \ni(y, s) \rightarrow(x, t)} v(y, s) \geq w(x, t)=u(x, t) \geq h(x, t) .
$$

Hence, the parabolic comparison principle in Theorem 2.4 shows that $h \leq v$ in $\widetilde{G}$, and thus $h \leq w$ in $Q_{t_{1}, t_{2}}$.

\section{Boundary regularity}

Definition 3.1. A boundary point $\xi_{0} \in \partial \Theta$ is regular with respect to $\Theta$, if

$$
\lim _{\Theta \ni \xi \rightarrow \xi_{0}} \bar{H} f(\xi)=f\left(\xi_{0}\right)
$$

whenever $f: \partial \Theta \rightarrow \mathbf{R}$ is continuous. Here $\bar{H} f$ denotes the upper Perron solution of $f$.

Observe that since $\underline{H} f=-\bar{H}(-f)$, regularity can equivalently be formulated using lower Perron solutions. In the following we will omit the explicit reference to $\Theta$, whenever no confusion may arise.

Our aim is next to characterise regular boundary points using families of barriers. Such a characterisation serves two purposes: to give a criterion for regularity, and to deduce various consequences of regularity. For the former, one would like to have as weak a condition as possible, whereas for the latter a stronger condition is often useful. Therefore, we introduce two conditions, which turn out to be equivalent.

Definition 3.2. Let $\xi_{0} \in \partial \Theta$. A family of functions $w_{j}: \Theta \rightarrow(0, \infty], j=1,2, \ldots$, is a barrier family in $\Theta$ at the point $\xi_{0}$ if for each $j$,

(a) $w_{j}$ is a positive $p$-superparabolic function in $\Theta$;

(b) $\lim _{\Theta \ni \zeta \rightarrow \xi_{0}} w_{j}(\zeta)=0$;

(c) for each $k=1,2, \ldots$, there is a $j$ such that

$$
\liminf _{\Theta \ni \zeta \rightarrow \xi} w_{j}(\zeta) \geq k \quad \text { for all } \xi \in \partial \Theta \text { with }\left|\xi-\xi_{0}\right| \geq 1 / k
$$

We also say that the family $w_{j}$ is a strong barrier family in $\Theta$ at the point $\xi_{0}$ if, in addition the following conditions hold:

(d) $w_{j}$ is continuous in $\Theta$; 
(e) there is a nonnegative function $d \in C(\bar{\Theta})$, with $d(z)=0$ if and only if $z=\xi_{0}$, such that for each $k=1,2, \ldots$, there is a $j=j(k)$ such that $w_{j} \geq k d$ in $\Theta$.

Note that in (c) the conditions on $w_{j}$ are only at $\partial \Theta$, while in (e) there is a requirement on $w_{j}$ in all of $\Theta$. The latter will be important when proving several of the consequences of the barrier characterisation that we derive later in this section. Note also that $(\mathrm{e}) \Rightarrow(\mathrm{c})$.

In classical potential theory, a barrier is a superharmonic (when dealing with the Laplace equation) or superparabolic (when dealing with the heat equation) function $w$ such that

$$
\lim _{\zeta \rightarrow \xi_{0}} w(\zeta)=0 \quad \text { and } \quad \liminf _{\zeta \rightarrow \xi} w(\zeta)>0 \text { for } \xi \in \partial \Theta \backslash\left\{\xi_{0}\right\}
$$

Existence of such a single barrier implies the regularity of a boundary point in the classical case, since one can scale and lift the barriers. However, this is not the case with the parabolic $p$-Laplacian, since the equation is not homogeneous with respect to $u$ : this reflects in that a scaled weak supersolution is not necessarily a weak supersolution. We think that it is precisely this lack of homogeneity, which forces the use of a whole family of barriers, instead of just simply one, but we do not know if a family is really required.

We are now ready to characterise regularity in terms of the existence of a barrier family (in our sense). At the same time, we show that the existence of a strong barrier family is equivalent. Note that we do not show that every barrier family is a strong barrier family, only that if there exists a barrier family then there also exists a strong barrier family.

Theorem 3.3. Let $\xi_{0} \in \partial \Theta$. Then the following are equivalent:

(1) $\xi_{0}$ is regular;

(2) there is a barrier family at $\xi_{0}$;

(3) there is a strong barrier family at $\xi_{0}$.

Proof. $(2) \Rightarrow(1)$ First we show that if there is a barrier family at $\xi_{0} \in \partial \Theta$, then $\xi_{0}$ is a regular boundary point. Since $f$ in Definition 3.1 is continuous, for each $\varepsilon>0$ there exists a constant $\delta>0$ such that if $\left|\xi-\xi_{0}\right|<\delta, \xi \in \partial \Theta$, then $\left|f(\xi)-f\left(\xi_{0}\right)\right|<\varepsilon$. We can therefore choose $j \geq 1$ large enough so that

$$
\liminf _{\Theta \ni \zeta \rightarrow \xi} w_{j}(\zeta)+\varepsilon+f\left(\xi_{0}\right)>f(\xi) \quad \text { for all } \xi \in \partial \Theta
$$

Thus $w_{j}+\varepsilon+f\left(\xi_{0}\right)$ belongs to the upper class $\mathcal{U}_{f}$, and hence

$$
\limsup _{\Theta \ni \zeta \rightarrow \xi_{0}} \bar{H} f(\zeta) \leq \lim _{\Theta \ni \zeta \rightarrow \xi_{0}} w_{j}(\zeta)+\varepsilon+f\left(\xi_{0}\right)=\varepsilon+f\left(\xi_{0}\right) .
$$

Since $-w_{j}-\varepsilon+f\left(\xi_{0}\right)$ is in the lower class (multiplication by -1 is allowed) if $j$ is large enough, we similarly obtain that

$$
\liminf _{\Theta \ni \zeta \rightarrow \xi_{0}} \bar{H} f(\zeta) \geq \liminf _{\Theta \ni \zeta \rightarrow \xi_{0}} \underline{H} f(\zeta) \geq-\varepsilon+f\left(\xi_{0}\right) .
$$

Letting $\varepsilon \rightarrow 0$ shows that $\xi_{0}$ is regular.

$(1) \Rightarrow(3)$. Next we prove that if $\xi \in \partial \Theta$ is regular, then there exists a strong barrier family at $\xi_{0}$.

Without loss of generality we may assume that $\xi_{0}$ is the origin. For $(x, t) \in \mathbf{R}^{n+1}$ we define

$$
d(x, t)=\frac{p-1}{p}|x|^{p /(p-1)}+\frac{n}{2 \operatorname{diam} \Theta} t^{2}
$$


and

$$
\psi_{j}(x, t)=j \frac{p-1}{p}|x|^{p /(p-1)}+j^{p-1} \frac{n}{2 \operatorname{diam} \Theta} t^{2} \geq \min \left\{j, j^{p-1}\right\} d(x, t) .
$$

A straightforward computation shows that in $\Theta$,

$$
\partial_{t} \psi_{j}-\Delta_{p} \psi_{j}=j^{p-1} \frac{n t}{\operatorname{diam} \Theta}-j^{p-1} n \leq 0
$$

i.e. $\psi_{j}$ is $p$-subparabolic. Setting $w_{j}:=\underline{H} \psi_{j}$ gives us a strong barrier family at $\xi_{0}$. Indeed, (e) in Definition 3.2 follows from the definition of the lower Perron solution, as it yields $w_{j} \geq \psi_{j}$, and (b) from the fact that $\xi_{0}$ is regular.

$(3) \Rightarrow(2)$ This is trivial.

The first consequence of the barrier characterisation is the following restriction theorem.

Proposition 3.4. Let $\xi_{0} \in \partial \Theta$ and let $G \subset \Theta$ be open and such that $\xi_{0} \in \partial G$. If $\xi_{0}$ is regular with respect to $\Theta$, then $\xi_{0}$ is regular with respect to $G$.

Proof. By Theorem 3.3, there is a strong barrier family $\left\{w_{j}\right\}_{j=1}^{\infty}$ in $\Theta$ at $\xi_{0}$. Let $d$ be as given in condition (e). Let also $w_{j}^{\prime}=\left.w_{j}\right|_{G}, j \geq 1$, and $d^{\prime}=\left.d\right|_{G}$. Then $\left\{w_{j}^{\prime}\right\}_{j=1}^{\infty}$ is a strong barrier family in $G$ at $\xi_{0}$, and thus Theorem 3.3 implies that $\xi_{0}$ is a regular boundary point with respect to $G$.

Another consequence of the barrier characterisation is that regularity is a local property.

Proposition 3.5. Let $\xi_{0} \in \partial \Theta$ and $B$ be a ball containing $\xi_{0}$. Then $\xi_{0}$ is regular with respect to $\Theta$ if and only if $\xi_{0}$ is regular with respect to $B \cap \Theta$.

Proof. Proposition 3.4 immediately implies that if $\xi_{0}$ is regular with respect to $\Theta$, then it is also regular with respect to $B \cap \Theta$.

Next we show that if $\xi_{0}$ is regular with respect to $B \cap \Theta$, then it is regular with respect to $\Theta$. By Theorem 3.3, there is a strong barrier family $\left\{w_{j}\right\}_{j=1}^{\infty}$ in $B \cap \Theta$, and a nonnegative continuous function $d$ associated with it. Let $m=\inf _{\bar{\Theta} \cap \partial B} d>0$,

$$
d^{\prime}=\left\{\begin{array}{ll}
\min \{d, m\} & \text { in } B \cap \bar{\Theta} \\
m & \text { in } \mathbf{R}^{n+1} \backslash B
\end{array} \quad \text { and } \quad w_{k}^{\prime}= \begin{cases}\min \left\{w_{j(k)}, k m\right\} & \text { in } B \cap \Theta \\
k m & \text { in } \Theta \backslash B\end{cases}\right.
$$

where $j(k)$ is as in Definition $3.2(\mathrm{e}), k=1,2, \ldots$.

Then $w_{k}^{\prime}$ is continuous in $\Theta$, satisfies $w_{k}^{\prime} \geq k d^{\prime}$ in $\Theta$, and by the pasting lemma (Lemma 2.9), $w_{k}^{\prime}$ is $p$-superparabolic in $\Theta$. Hence $\left\{w_{k}^{\prime}\right\}_{k=1}^{\infty}$ is the desired strong barrier family in $\Theta$ at $\xi_{0}$, and this implies that $\xi_{0}$ is regular with respect to $\Theta$.

Next we state one of our main results. As we have already remarked, if $u$ is a (super)solution to the $p$-parabolic equation and $\gamma \geq 0$, then in general $\gamma u$ is not a (super)solution to the same equation, except when $p=2$. Instead, $\gamma u$ is a solution to a multiplied $p$-parabolic equation, namely to

$$
a \frac{\partial u}{\partial t}=\Delta_{p} u, \quad a=\gamma^{p-2}
$$

as is apparent by straightforward calculations. This fact makes it possible to show that when $p \neq 2$, the regular points are the same for all multiplied $p$-parabolic equations. This is quite surprising, because a similar statement is known to be false 
for the heat equation as a direct consequence of Petrovskilı's criterion discussed in the introduction.

On the other hand, for $p=2$ it is enough to have one barrier to get regularity, since any positive multiple of a barrier is still a barrier. So the gist of the argument is the following: for $p=2$ one barrier is enough, but multiplied equations have different regular points, whereas for $p \neq 2$ a family of barriers is required (we believe), but a regular point is such for all multiplied equations.

Theorem 3.6. Let $\xi_{0} \in \partial \Theta$ and $a>0$. If $p \neq 2$, then $\xi_{0}$ is regular if and only if it is regular with respect to the multiplied p-parabolic equation

$$
a \frac{\partial u}{\partial t}=\Delta_{p} u
$$

Proof. Let $w$ be a weak supersolution to the $p$-parabolic equation and let $\widetilde{w}=$ $a^{1 /(p-2)} w$. Then

$$
a \partial_{t} \widetilde{w}-\Delta_{p} \widetilde{w}=a^{1+1 /(p-2)} \partial_{t} w-a^{1+1 /(p-2)} \Delta_{p} w \geq 0
$$

and thus $\widetilde{w}$ is a weak supersolution to the multiplied $p$-parabolic equation, and vice versa. The same equivalence obviously holds also for $p$-superparabolic functions.

It follows directly that $u \in \mathcal{U}_{f}$ if and only if $a^{1 /(p-2)} u \in \mathcal{U}_{a^{1 /(p-2)} f}^{a}$, where $\mathcal{U}_{f}^{a}$ is the upper class defining the upper Perron solution with respect to (3.2). The equivalence of regularity of $\xi_{0}$ with respect to (2.1) and with respect to (3.2) now follows directly from the definition.

It is noteworthy that Theorem 3.6 holds both for $p>2$ and $1<p<2$. The next corollary immediately follows from the proof of the previous result.

Corollary 3.7. Let $f \in C(\partial \Theta)$. Then

$$
\bar{H}^{a}\left(a^{1 /(p-2)} f\right)=a^{1 /(p-2)} \bar{H} f,
$$

where $\bar{H}^{a}$ denotes the upper Perron solution with respect to (3.2).

Lemma 3.8. Assume that $\Theta_{1}, \ldots, \Theta_{m}$ are pairwise disjoint bounded open sets in $\mathbf{R}^{n+1}$ with $\xi_{0} \in \partial \Theta_{j}, j=1, \ldots, m$. Then $\xi_{0}$ is regular with respect to $\Theta=\bigcup_{j=1}^{m} \Theta_{j}$ if and only if it is regular with respect to each $\Theta_{j}, j=1, \ldots, m$.

Proof. The necessity follows from Proposition 3.4. As for the sufficiency, let $f \in$ $C(\partial \Theta)$. Then $\left.(\bar{H} f)\right|_{\Theta_{j}}=\left.\bar{H}_{\Theta_{j}} f\right|_{\partial \Theta_{j}}$ and thus

$$
\lim _{\Theta_{j} \ni \xi \rightarrow \xi_{0}} \bar{H} f(\xi)=\left.\lim _{\Theta_{j} \ni \xi \rightarrow \xi_{0}} \bar{H}_{\Theta_{j}} f\right|_{\partial \Theta_{j}}(\xi)=f\left(\xi_{0}\right)
$$

for $j=1, \ldots, m$. It follows that

$$
\lim _{\Theta \ni \xi \rightarrow \xi_{0}} \bar{H} f(\xi)=f\left(\xi_{0}\right)
$$

and hence $\xi_{0}$ is regular with respect to $\Theta$.

Next we let $\Theta=G_{T}=G \times(0, T)$, where $G \subset \mathbf{R}^{n}$ is an open set, and recall two results from Kilpeläinen-Lindqvist [21]. As we want to use our barrier family characterisation, we sketch the proofs in this context for the convenience of the reader.

Theorem 3.9. Let $x_{0} \in \partial G$ and $0<t_{0} \leq T$. Then the boundary point $\xi_{0}=\left(x_{0}, t_{0}\right)$ is regular with respect to $G_{T}$, in the sense of Definition 3.1, if and only if $x_{0}$ is regular for $p$-harmonic functions with respect to $G$. 
A $p$-harmonic function is a continuous weak solution to the (elliptic) p-Laplace equation $\Delta_{p} u=0$.

Proof. The proof of Theorem 6.5 in [21] can immediately be modified to use the barrier family characterisation. Suppose that $x_{0}$ is regular for $p$-harmonic functions with respect to $G$ (for more details on this notion, see for example HeinonenKilpeläinen-Martio [18]). Let $\varphi(x)=\left|x-x_{0}\right|$ and let $u_{j}$ be a solution to

$$
\left\{\begin{array}{l}
\Delta_{p} u_{j}=-j^{p-1} \\
u_{j}-j \varphi \in W_{0}^{1, p}(G) .
\end{array} \quad \text { in } G,\right.
$$

Then $u_{j}$ is $p$-superharmonic and $u_{j}(x) \geq j\left|x-x_{0}\right|$ because $\varphi$ is $p$-subharmonic. Define

$$
w_{j}(x, t)=u_{j}(x)+j^{p-1}\left(t_{0}-t\right) .
$$

Then

$$
\Delta_{p} w_{j}=-j^{p-1}=\partial_{t} w_{j},
$$

and it follows that $\left\{w_{j}\right\}_{j=1}^{\infty}$ is the desired barrier family, and $\xi_{0}$ is regular with respect to $G_{T}$.

The other direction of the proof of Theorem 6.5 in [21] holds verbatim.

We will also need the following result, stating that what happens in the future does not affect the regularity of the boundary point. To be more precise, if we split the domain $\Theta$ at the level $t_{0}$, and consider a boundary point $\left(x_{0}, t_{0}\right)$ at the same time instant, then the lower part

$$
\Theta_{-}=\left\{(x, t) \in \Theta: t<t_{0}\right\}
$$

determines the regularity. We begin with an introductory lemma, which we will also use later on. In particular, it shows that the earliest points are always regular.

Lemma 3.10. Let $\xi_{0}=\left(x_{0}, t_{0}\right) \in \partial \Theta$. If $\xi_{0} \notin \partial \Theta_{-}\left(\right.$in particular, if $\left.\Theta_{-}=\varnothing\right)$, then $\xi_{0}$ is regular.

Proof. If $\xi_{0} \notin \partial \Theta_{-}$, then the functions

$$
f_{j}(x, t)=j \frac{p-1}{p}\left|x-x_{0}\right|^{p /(p-1)}+n j^{p-1}\left(t-t_{0}\right)
$$

are $p$-parabolic in $\mathbf{R}^{n+1}$ and form a strong barrier family in $\Theta \cap V$ for some neighbourhood $V$ of $\xi_{0}$.

It follows from Theorem 3.9 and Lemma 3.10 that in the setting of Theorem 2.3, the Perron solution coincides with the one provided by Theorem 2.3.

Theorem 3.11. Let $\xi_{0}=\left(x_{0}, t_{0}\right) \in \partial \Theta$ and $\Theta_{-} \neq \varnothing$. Then $\xi_{0}$ is regular with respect to $\Theta$ if and only if either $\xi_{0}$ is regular with respect to $\Theta_{-}$or $\xi_{0} \notin \partial \Theta_{-}$.

Proof. Suppose that $\xi_{0}$ is a regular boundary point with respect to $\Theta$. Then by Proposition 3.4, either $\xi_{0} \notin \partial \Theta_{-}$or $\xi_{0}$ is also regular with respect to $\Theta_{-}$.

To prove the converse, due to Lemma 3.10, we may assume that $\xi_{0} \in \partial \Theta_{-}$is a regular boundary point of $\Theta_{-}$. Let $w_{j}=\underline{H} \psi_{j}$, where $\psi_{j}$ are as in (3.1). As in [21], it can be shown that the restriction to $\Theta_{-}$of $w_{j}$ is the upper Perron solution of $\psi_{j}$ in $\Theta_{-}$, and hence

$$
\lim _{\Theta_{-} \ni \xi \rightarrow \xi_{0}} w_{j}(\xi)=\psi_{j}\left(\xi_{0}\right)=0 .
$$


This also implies that $w_{j}$, extended by $\psi_{j}$ to $\partial \Theta$, is continuous at $\xi_{0}$. Again following [21], it can be shown that the restriction to $\Theta_{+}=\left\{(x, t) \in \Theta: t>t_{0}\right\}$ of $w_{j}$ coincides with the lower Perron solution of (the above extension) $w_{j}$ with respect to $\Theta_{+}$. Since earliest points are regular by Lemma 3.10, we can conclude from this that also

$$
\lim _{\Theta \backslash \Theta-\ni \xi \rightarrow \xi_{0}} w_{j}(\xi)=0
$$

showing that $w_{j}$ form a barrier family in $\Theta$.

The following characterisation is a bit similar in flavour to our barrier characterisation, in that it deduces regularity from properties of a countable family.

Proposition 3.12. Let $\xi_{0} \in \partial \Theta$ and let $d \in C(\partial \Theta)$ be such that $d\left(\xi_{0}\right)=0$ and $d(\xi)>0$ for $\xi \in \partial \Theta \backslash\left\{\xi_{0}\right\}$. Then $\xi_{0}$ is regular if and only if

$$
\lim _{\Theta \ni \xi \rightarrow \xi_{0}} \bar{H}(j d)(\xi)=0 \quad \text { for } j=1,2, \ldots
$$

A typical example is $d(\xi)=\left|\xi-\xi_{0}\right|^{\alpha}$ with $\alpha>0$. In the elliptic $p$-harmonic case, a similar characterisation was given by Björn-Björn [3], Theorem 4.2 and Remarks 6.2 (which can also be found as Theorem 11.2 and Remark 11.12 in [4]). In the $p$-harmonic case only the limit of $\bar{H} d$ is required, and the same is also true for the heat equation. We do not know whether one limit is sufficient in the $p$-parabolic case, and this issue seems closely related to the problem of whether one barrier is sufficient.

A corresponding characterisation (with a family of Perron solutions) was recently given for the elliptic variable exponent $p(\cdot)$-harmonic functions by AdamowiczBjörn-Björn [1], Theorem 7.1.

Proof. The necessity is obvious. For the sufficiency, let $f \in C(\partial \Theta)$ and $\varepsilon>0$. Then we can find $j$ such that $f<j d+f\left(\xi_{0}\right)+\varepsilon$ on $\partial \Theta$. Thus,

$$
\lim _{\Theta \ni \xi \rightarrow \xi_{0}} \bar{H} f(\xi) \leq f\left(\xi_{0}\right)+\varepsilon+\lim _{\Theta \ni \xi \rightarrow \xi_{0}} \bar{H}(j d)(\xi)=f\left(\xi_{0}\right)+\varepsilon .
$$

Letting $\varepsilon \rightarrow 0$ shows that $\lim _{\Theta \ni \xi \rightarrow \xi_{0}} \bar{H} f(\xi) \leq f\left(\xi_{0}\right)$. Applying this also to $-f$ yields that $\lim _{\Theta \ni \xi \rightarrow \xi_{0}} \bar{H} f(\xi)=f\left(\xi_{0}\right)$, and thus $\xi_{0}$ is regular.

\section{The exterior ball condition}

In this section, we will show that if the domain satisfies an exterior ball condition, then it is regular. However, the "south and north poles" have to be excluded. To be more precise, the condition $x_{1} \neq 0$ in Proposition 4.1 below is meant to exclude both the south pole $\left(x_{1}, t_{1}-R_{1}\right)$ and the north pole $\left(x_{1}, t_{1}+R_{1}\right)$ of the exterior ball as a tangent point. The restriction on the southern pole was already pointed out by Kilpeläinen and Lindqvist (see the comment at the end of the proof of Theorem 6.2 in [21]). Indeed, the top of a cylindrical domain gives a natural counterexample. As for the north pole, when $x$ is close to $x_{1}=0, \Delta_{p} u$ is positive, and, curiously enough, the argument does not work.

Since the space is homogeneous and the $p$-parabolic equation is translation invariant, the geometric conditions implying regularity are the same at all points. We therefore describe conditions for regularity of the origin from now on.

Proposition 4.1. (Exterior ball condition) Let $\xi_{0}=(0,0) \in \partial \Theta$. Suppose that there exists a ball $B_{1}=B\left(\xi_{1}, R_{1}\right)$, with centre $\xi_{1}=\left(x_{1}, t_{1}\right)$ and radius $R_{1}$, such that $B_{1} \cap \Theta=\varnothing$ and $\xi_{0} \in \partial B_{1} \cap \partial \Theta$. If $x_{1} \neq 0$ then $\xi_{0}$ is regular with respect to $\Theta$. 
Proof. Since the case $p=2$ is classical, we assume that $p \neq 2$. We follow the ideas introduced in Kilpeläinen-Lindqvist [21].

Let $\xi_{2}=\left(x_{2}, t_{2}\right)=\frac{1}{2} \xi_{1}$ and $R_{2}=\frac{1}{2} R_{1}$. Note that $R_{1}=\left|\xi_{1}\right|$ and $R_{2}=\left|\xi_{2}\right|$. Let $\delta=\frac{1}{2}\left|x_{2}\right|>0$ and $\Theta_{0}=\Theta \cap B\left(\xi_{0}, \delta\right)$. Here we have used that $x_{1} \neq 0$.

Let $\xi=(x, t) \in \bar{\Theta}_{0}$ and $R=\left|\xi-\xi_{2}\right| \leq 2 R_{2}$. Also let $j_{0} \geq(n+p-2) /(p-1) \delta^{2}$ and $j \geq j_{0}$ be integers. Define

$$
w_{j}(\xi)=\gamma\left(e^{-j R_{2}^{2}}-e^{-j R^{2}}\right),
$$

where $\gamma=\gamma(j)>0$ will be chosen later. As in [21], easy calculations yield

$$
\partial_{t} w_{j}=2 j \gamma e^{-j R^{2}}\left(t-t_{2}\right) \geq-4 j \gamma R_{2} e^{-j R^{2}}
$$

and

$$
\begin{aligned}
\Delta_{p} w_{j} & =(2 j \gamma)^{p-1}\left|x-x_{2}\right|^{p-2} e^{-j(p-1) R^{2}}\left[n+p-2-2 j(p-1)\left|x-x_{2}\right|^{2}\right] \\
& \leq(2 j \gamma)^{p-1}\left|x-x_{2}\right|^{p-2} e^{-j(p-1) R^{2}}\left[n+p-2-2 j(p-1) \delta^{2}\right] .
\end{aligned}
$$

The choice of $j$ implies that $n+p-2-2 j(p-1) \delta^{2} \leq-j(p-1) \delta^{2}$. Since $\delta \leq$ $\left|x-x_{2}\right| \leq R \leq 2 R_{2}$, this gives

$$
\begin{aligned}
\Delta_{p} w_{j} & \leq-(2 j \gamma)^{p-1}\left|x-x_{2}\right|^{p-2} j(p-1) \delta^{2} e^{-j(p-1) R^{2}} \\
& \leq-C_{0}(2 j \gamma)^{p-1} j(p-1) e^{-j(p-1) R^{2}}
\end{aligned}
$$

where

$$
C_{0}= \begin{cases}\left(2 R_{2}\right)^{p-2} \delta^{2}, & 1<p<2, \\ \delta^{p}, & p>2 .\end{cases}
$$

In order to have $\Delta_{p} w_{j} \leq \partial_{t} w_{j}$ it is enough to verify that

$$
4 j \gamma R_{2} e^{-j R^{2}} \leq C_{0}(2 j \gamma)^{p-1} j(p-1) e^{-j(p-1) R^{2}},
$$

which is equivalent to

$$
\gamma^{p-2} \geq \frac{j^{1-p} R_{2} e^{j(p-2) R^{2}}}{2^{p-3}(p-1) C_{0}}=: C_{1} j^{1-p} e^{j(p-2) R^{2}},
$$

where $C_{1}=R_{2} / 2^{p-3}(p-1) C_{0}$. Choose now

$$
\gamma=\gamma(j)= \begin{cases}\left(C_{1} j^{1-p}\right)^{1 /(p-2)} e^{j R_{2}^{2}}, & 1<p<2, \\ \left(C_{1} j^{1-p}\right)^{1 /(p-2)} e^{4 j R_{2}^{2}}, & p>2 .\end{cases}
$$

As $B\left(\xi_{2}, R_{2}\right) \cap \Theta_{0}$ is empty, we have that $R_{2} \leq R \leq 2 R_{2}$, which shows that (4.1) holds. Therefore, $w_{j}$ is $p$-superparabolic in $\Theta_{0}$.

We next want to show that $w_{j}$ satisfies (c) of Definition 3.2, and thus that $\left\{w_{j}\right\}_{j=j_{0}}^{\infty}$ is a barrier family, condition (b) being immediate.

Let $\beta$ be the angle between the vectors $-\xi_{1}$ and $\xi-\xi_{1}, r_{0}=|\xi|$ and $r_{1}=$ $\left|\xi-\xi_{1}\right| \geq R_{1}$. The cosine theorem yields $r_{0}^{2}=r_{1}^{2}+R_{1}^{2}-2 r_{1} R_{1} \cos \beta$. Together with the cosine theorem again and the inequality $r_{1} \geq R_{1}$ this yields

$$
\begin{aligned}
R^{2}-R_{2}^{2} & =\left(\frac{1}{2} R_{1}\right)^{2}+r_{1}^{2}-r_{1} R_{1} \cos \beta-\left(\frac{1}{2} R_{1}\right)^{2} \\
& =r_{1}^{2}-\frac{1}{2}\left(r_{1}^{2}+R_{1}^{2}-r_{0}^{2}\right)=\frac{1}{2} r_{1}^{2}-\frac{1}{2} R_{1}^{2}+\frac{1}{2} r_{0}^{2} \geq \frac{1}{2} r_{0}^{2} .
\end{aligned}
$$

It follows that for $\xi \in \bar{\Theta}_{0} \backslash B\left(\xi_{0}, r\right)$,

$$
w_{j}(\xi)=\gamma e^{-j R_{2}^{2}}\left(1-e^{j\left(R_{2}^{2}-R^{2}\right)}\right) \geq \gamma e^{-j R_{2}^{2}}\left(1-e^{-j r^{2} / 2}\right) .
$$


Inserting the expression (4.2) for $\gamma$ we obtain

$$
w_{j}(\xi) \geq \begin{cases}\left(C_{1} j^{1-p}\right)^{1 /(p-2)}\left(1-e^{-j r^{2} / 2}\right), & 1<p<2, \\ \left(C_{1} j^{1-p}\right)^{1 /(p-2)} e^{3 j R_{2}^{2}}\left(1-e^{-j r^{2} / 2}\right), & p>2 .\end{cases}
$$

For a fixed $r$, the right-hand sides tend to $\infty$, as $j \rightarrow \infty$, showing that $\left\{w_{j}\right\}_{j=j_{0}}^{\infty}$ is a barrier family with respect to $\Theta_{0}$. Thus, by Theorem $3.3, \xi_{0}=(0,0)$ is regular with respect to $\Theta_{0}$, and hence, by Proposition 3.5, it is regular with respect to $\Theta$.

\subsection{Regularity at the "north pole"}

The proof above for the exterior ball condition does not work when $\xi_{0}=(0,0)$ is the north pole of the ball. Here we discuss some simple sufficient conditions for the regularity of $\xi_{0}$, when it can be considered as some sort of north pole for proper sets touching $\partial \Theta$ from below.

Observe that if we have a flat bottom, i.e. the half-space $\{(x, t): t<0\} \subset$ $\mathbf{R}^{n+1} \backslash \Theta$, then Lemma 3.10 gives the regularity.

Proposition 4.2. Let $\Theta \subset \mathbf{R}^{n+1}$ be an open set and $(0,0) \in \partial \Theta$. Assume that for some $\theta>0$,

$$
\Theta \subset\left\{(x, t): t>-\theta|x|^{l}\right\}
$$

where $l \geq p /(p-1)$ if $1<p<2$, and $l>p$ if $p>2$. Then $(0,0)$ is regular with respect to $\Theta$.

Proof. By Propositions 3.4 and 3.5 and Theorem 3.11 we may assume that

$$
\Theta=\left\{(x, t): t>-\theta|x|^{l} \text { and }-1<t<0\right\} .
$$

We localise the problem by considering

$$
G^{j}=\left\{(x, t):|x|<\frac{1}{j^{1 / k}} \text { and }-\frac{\theta}{j^{l / k}}<t<0\right\} \subset \Theta,
$$

where $k>0$ will be fixed later. Notice that the set $G^{j}$ gets smaller, as $j$ grows. Now let

$$
f_{j}(x, t)=j \frac{p-1}{p}|x|^{p /(p-1)}+n j^{p-1} t,
$$

which is $p$-parabolic in $\mathbf{R}^{n+1}$. If $1<p<2$, then $f_{j}$ is positive in $G^{j} \cap \Theta$, provided $l \geq p /(p-1)$ and $j$ is large enough. If $p>2$, then $f_{j}$ is positive in $G^{j} \cap \Theta$, provided $l>p /(p-1)+k(p-2)$ and $j$ is large enough. Define

$$
m_{j}:=\inf _{\Theta \cap \partial G^{j}} f_{j}=j \frac{p-1}{p}\left(\frac{1}{j^{1 / k}}\right)^{p /(p-1)}-n j^{p-1} \frac{\theta}{j^{l / k}} .
$$

We want $m_{j} \rightarrow \infty$ as $j \rightarrow \infty$, and this happens if

$$
1-\frac{p}{(p-1) k}>0 \text { and } 1-\frac{p}{(p-1) k}>p-1-\frac{l}{k} .
$$

The first condition is satisfied if $k>p /(p-1)$, and the second condition is satisfied if $l>p /(p-1)+k(p-2)$. This holds for $1<p<2, l \geq p /(p-1)$ and all $k>p /(p-1)$, whereas for $p \geq 2$ and $l>p$, this is true, provided we choose $k$ sufficiently close to $p /(p-1)$.

Now let

$$
h_{j}= \begin{cases}\min \left\{f_{j}, m_{j}\right\} & \text { in } G^{j} \\ m_{j} & \text { in } \Theta \backslash G^{j}\end{cases}
$$


Since $f_{j}$ is $p$-parabolic in $\mathbf{R}^{n+1}$, the pasting lemma (Lemma 2.9), applied to $m_{j}$ and $\left.f_{j}\right|_{G^{j}}$, shows that $h_{j}$ is a positive (continuous) $p$-superparabolic function in $\Theta$, if $j$ is large enough. As $m_{j} \rightarrow \infty$ and $G^{j}$ shrinks to $(0,0)$, it follows that $\left\{h_{j}\right\}_{j=m}^{\infty}$ is a strong barrier family if $m$ is large enough, and thus $(0,0)$ is regular with respect to $\Theta$.

\section{Exterior cone conditions}

Let us consider $\xi_{0} \in \partial \Theta$. Without loss of generality we assume that $\xi_{0}=(0,0)$. Assume that there is an open cone $C$ in the exterior $\mathbf{R}^{n+1} \backslash \Theta$ with vertex at $\xi_{0}$. When dealing with a space-time cylindrical domain, it is well-known that the nonparabolic part of the boundary is irregular; therefore, it is necessary that the cone $C$ contains some point $\left(x_{C}, t_{C}\right)$ with $t_{C} \leq 0$ (or equivalently a point with $\left.t_{C}<0\right)$ in order to have a chance of implying the regularity of $\xi_{0}$.

As regularity is a local property by Proposition 3.5, we assume that the full cone is in the exterior, and we may therefore assume that $\left|\left(x_{C}, t_{C}\right)\right|=1$. Since $C$ is open, we can find an open cylindrical subcone $C_{0}$ with vertex $\xi_{0}$ containing $\left(x_{C}, t_{C}\right)$ and such that $\bar{\Theta} \cap \bar{C}_{0}=\left\{\xi_{0}\right\}$.

To work out concrete examples to begin with, we show in Proposition 5.1 that the exterior cone condition in $\mathbf{R}^{1+1}$ yields regularity for $1<p<\infty$. We then use this to obtain a more general criterion ensuring regularity in the $1+1$-dimensional case, see Theorem 5.2. Then in Section 5.2, we focus on the higher-dimensional case.

\subsection{Exterior cone condition in $\mathbf{R}^{1+1}$ for $1<p<\infty$}

Proposition 5.1. (Exterior cone condition in $1+1$ dimensions) Let $\Theta \subset \mathbf{R}^{1+1}$ be bounded and $\xi_{0}=(0,0) \in \partial \Theta$. Assume that there is an open cone $C \subset \mathbf{R}^{1+1} \backslash \Theta$ with $\xi_{0}$ as vertex, and a point $\xi=\left(x_{C}, t_{C}\right) \in C$ with $t_{C} \leq 0$. Then $\xi_{0}$ is regular.

Case 1 below follows from the exterior ball condition or from Proposition 5.3, and so only Case 2 is actually needed. However, because Case 1 is more elementary here and provides concrete examples of simple barrier families, we have decided to include it here.

Proof. We may assume that the cone $C$ is a full cylindrical cone, that $\Theta \subset B\left(\xi_{0}, 1\right)$ and that $\bar{C} \cap \bar{\Theta}=\left\{\xi_{0}\right\}$. By Theorem 3.11, we may also assume that $\Theta \subset\{(x, t)$ : $t<0\}$.

Case 1. Horizontal cone, i.e. $t_{C}=0$, see Figure 1 . We may assume that $x_{C}=1$, and thus

$$
C=\{(x, t):|t|<\gamma x\} .
$$

where $\gamma$ is a positive parameter. Let

$$
u_{\mu, \alpha}(x, t)=\mu\left(1-e^{-\alpha(|t|-\gamma x)}\right)
$$

where $\mu$ and $\alpha$ are positive numbers. We want $u_{\mu, \alpha}$ to be $p$-superparabolic in $\Theta$. We see that

$$
\partial_{x} u_{\mu, \alpha}=-\mu \alpha \gamma e^{-\alpha(|t|-\gamma x)}
$$

and hence

$$
\begin{aligned}
\Delta_{p} u_{\mu, \alpha} & =-(\mu \alpha \gamma)^{p-1} \frac{\partial}{\partial x} e^{-(p-1) \alpha(|t|-\gamma x)} \\
& =-(\mu \alpha \gamma)^{p-1}(p-1) \alpha \gamma e^{-(p-1) \alpha(|t|-\gamma x)}<0 \text { in } \Theta .
\end{aligned}
$$




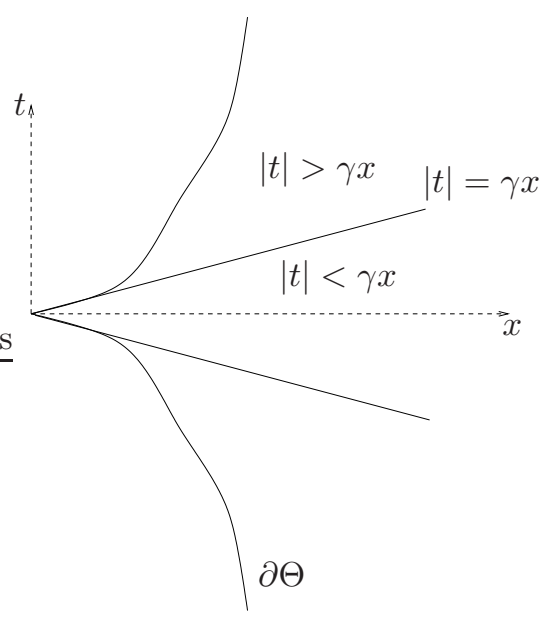

Figure 1. The exterior cone condition with a horizontal cone.

On the other hand,

$$
\partial_{t} u_{\mu, \alpha}=-\mu \alpha e^{-\alpha(|t|-\gamma x)} \quad \text { in } \Theta,
$$

since $t<0$. Thus, we have $\Delta_{p} u_{\mu, \alpha} \leq \partial_{t} u_{\mu, \alpha}$ if and only if

$$
-(\mu \alpha \gamma)^{p-1}(p-1) \alpha \gamma e^{-(p-1) \alpha(|t|-\gamma x)} \leq-\mu \alpha e^{-\alpha(|t|-\gamma x)}
$$

which is equivalent to

$$
\mu^{p-2} \alpha^{p-1} \gamma^{p}(p-1) \geq e^{(p-2) \alpha(|t|-\gamma x)} .
$$

As before, we consider $p>2$ and $1<p<2$ separately, starting with the former case, as it turns out to be simpler.

Case $1 a . p>2$. In this case we let $\alpha=1$ and thus $u_{\mu, 1}$ is $p$-superparabolic in $\Theta$ if

$$
\mu \geq \frac{e^{1+\gamma}}{\left(\gamma^{p}(p-1)\right)^{1 /(p-2)}}=: \mu_{0},
$$

since $\Theta \subset B\left(\xi_{0}, 1\right)$. It follows that for $j \geq \mu_{0}, w_{j}=u_{j, 1}$ is a suitable barrier family and thus $\xi_{0}$ is regular.

Case 1b. $1<p<2$. In this case we let $\alpha(\mu):=\left(\mu^{(2-p)} / \gamma^{p}(p-1)\right)^{1 /(p-1)}$. Then (5.1) is satisfied with $\alpha:=\alpha(\mu)$. Moreover, indexing with $j$ we have that $w_{j}:=u_{j, \alpha(j)}$ is $p$-superparabolic in $\Theta$. It also has the necessary limits at the boundary. Finally, for sufficiently large $j$, we have $\alpha(j) \geq 1$ and hence for $(x, t) \in \bar{\Theta}$,

$$
w_{j}(x, t)=j\left(1-e^{-\alpha(j)(|t|-\gamma x)}\right) \geq j\left(1-e^{-(|t|-\gamma x)}\right),
$$

showing that $w_{j}$ is a suitable barrier family, and thus $\xi_{0}$ is regular.

Case 2. Downwards cone, i.e. $t_{C}<0$, see Figure 2. (By downwards we do not mean straight downwards, i.e. $x_{C}$ is not necessarily 0 .)

In this 2-dimensional situation the cone splits $\Theta$ into two parts, one to the left and one to the right of the cone, or more formally into

$$
\begin{aligned}
& \Theta_{1}=\{(x, t) \in \Theta: x<y \text { whenever }(y, t) \in C\}, \\
& \Theta_{2}=\{(x, t) \in \Theta: x>y \text { whenever }(y, t) \in C\} .
\end{aligned}
$$




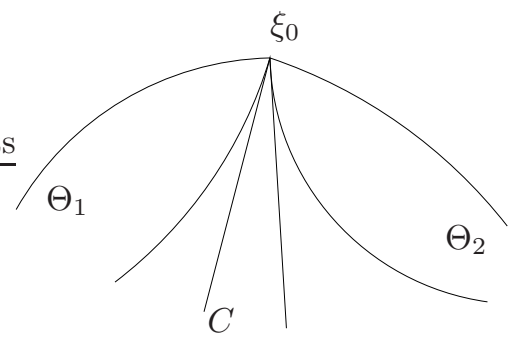

Figure 2. The exterior cone condition with a downwards cone.

It is possible that one of these is empty, but not both.

It is easy to see that $\Theta_{1}$, if nonempty, satisfies the exterior cone condition with a horizontal cone around $\xi_{0}$, and hence $\xi_{0}$ is regular with respect to $\Theta_{1}$ by Case 1, and similarly with respect to $\Theta_{2}$. It thus follows from Lemma 3.8 that $\xi_{0}$ is regular with respect to $\Theta$. (If $\Theta_{1}=\varnothing$ or $\Theta_{2}=\varnothing$ this follows directly.)

We can considerably generalise the previous result.

Theorem 5.2. Let $C=\{(x, t):|x| \leq-\theta t\} \subset \mathbf{R}^{1+1}$ for some $\theta>0$, and assume that $\xi_{0}=(0,0) \in \partial \Theta$ and that there is a closed nonsingleton connected set $A \subset C \backslash \Theta$ containing $\xi_{0}$. Then $\xi_{0}$ is regular.

Proof. Let $\left(x_{A}, t_{A}\right) \in A \backslash\left\{\xi_{0}\right\}$ so that $t_{A}<0$. Since regularity is a local property, we may assume that $\Theta \subset B\left(\xi_{0},-t_{A}\right)$. By Theorem 3.11, we may assume that $\Theta \subset\{(x, t): t<0\}$. We next split $\Theta$ into two parts,

$$
\begin{aligned}
& \Theta_{1}=\{(x, t) \in \Theta: x<y \text { whenever }(y, t) \in A\}, \\
& \Theta_{2}=\Theta \backslash \Theta_{1} .
\end{aligned}
$$

It is possible that one of these is empty, but not both. We can now conclude the proof exactly as in the proof of Case 2 of Proposition 5.1.

\subsection{Generalised horizontal cone condition in $\mathbf{R}^{n+1}$}

In this section we will show that if the lower half of a horizontal cone is in the complement then the point is regular.

Proposition 5.3. (Horizontal cone condition) Let $\Theta \subset \mathbf{R}^{n+1}$ be an open set and $\xi_{0}=(0,0) \in \partial \Theta$. If there exist $\theta, r>0$ and a unit vector $v \in \mathbf{R}^{n}$ such that the cone

$$
C:=\{(x, t):|(x, t)|<\theta x \cdot v, t \leq 0 \text { and }|x|<r\} \subset \mathbf{R}^{n+1} \backslash \Theta,
$$

then $\xi_{0}$ is regular with respect to $\Theta$.

Relying on potential theoretic tools we can deduce a fairly more general result, see Proposition 5.4 below. Proposition 5.3 then follows directly as a special case, by taking $\eta(t)=\gamma t$ and $E=\left\{x \in \mathbf{R}^{n}:|x|<\theta^{\prime} x \cdot v\right\}$ for some sufficiently small $\gamma, \theta^{\prime}>0$, and upon observing that the corresponding set $E^{\prime}$ defined by (5.3) below is then contained in the cone $C$ in (5.2).

Proposition 5.3 holds for any $p>1$, and in particular for $p=2$. It is immediate to see that a horizontal cone $C$ such as defined by (5.2), always contains a so-called tusk, that is a set in $\mathbf{R}^{n+1}$ of the form

$$
V:=\left\{(x, t):-T<t<0 \text { and }\left|x-(-t)^{1 / 2} x_{0}\right|^{2}<R^{2}(-t)\right\},
$$


for some $x_{0} \in \mathbf{R}^{n} \backslash\{0\}$, and positive constants $R$ and $T$, provided $T$ is small enough. Consider $\Theta \in \mathbf{R}^{n+1}$ and $\xi_{0}=(0,0) \in \partial \Theta$ : it is well known that if there is a tusk $V$ with $\bar{V} \cap \bar{\Theta}=\left\{\xi_{0}\right\}$, then $\xi_{0}$ is regular for the heat equation (see Effros-Kazdan [12], which refers to $\xi_{0}$ as being parabolically touchable, and Lieberman [31]). Therefore, under this point of view, Proposition 5.3 gives a weaker condition. We do not know, whether a proper tusk condition holds for the parabolic $p$-Laplacian, when $p \neq 2$.

Recall that for $1<p \leq n$ a set $A \subset \mathbf{R}^{n}$ is $p$-thick at $0 \in \mathbf{R}^{n}$ if

$$
\int_{0}^{1}\left(\frac{\operatorname{cap}_{p}\left(A \cap B^{\prime}(0, r), B^{\prime}(0,2 r)\right)}{r^{n-p}}\right)^{1 /(p-1)} \frac{d r}{r}<\infty,
$$

and $p$-thin otherwise, where for a given set $E \subseteq B^{\prime}(0,2 r), \operatorname{cap}_{p}\left(E, B^{\prime}(0,2 r)\right)$ is the variational $p$-capacity of $E$, and $B^{\prime}(0, r)$ is the ball of centre 0 and radius $r$ in $\mathbf{R}^{n}$.

The Wiener criterion says that 0 is regular for $p$-harmonic functions with respect to an open set $V$ if and only if $\mathbf{R}^{n} \backslash V$ is $p$-thick at 0 . It was obtained by Wiener [42] for $p=2$ in $\mathbf{R}^{3}$. In the nonlinear case, the sufficiency was obtained by Maz'ya [37] and Gariepy-Ziemer [16], and the necessity by Lindqvist and Martio [34] (for $p \geq$ $n-1$ ) and by Kilpeläinen and Malý [22], see also Chapter 4 of Malý-Ziemer [36]. These results have later been proved in more general settings in e.g. [38], [7], [5] and $[6]$.

Note that for $p>n$, by the same criterion, a singleton is always regular, which is useful and reflected in Example 5.5 below.

Proposition 5.4. Let $\Theta \subset \mathbf{R}^{n+1}$ be an open set and $\xi_{0}=(0,0) \in \partial \Theta$. Also let $E \subset \mathbf{R}^{n}$ be a closed set which is $p$-thick at 0 . Assume that for some $\theta>0$, some $\zeta \in \mathbf{R}^{n}$ with $|\zeta|=1$, and a nondecreasing differentiable function $\eta:\left[0, r_{0} / \theta\right] \rightarrow$ $[0, \infty)$, such that $\eta(0)=0$ and $\eta^{\prime} \leq \theta$ in $\left[0, r_{0} / \theta\right]$, we have

$$
E^{\prime}:=\left\{(x, t) \in B\left(\xi_{0}, r_{0}\right):-\frac{r_{0}}{\theta}<t<0 \text { and } x-\eta(-t) \zeta \in E\right\} \subset B\left(\xi_{0}, r_{0}\right) \backslash \Theta \text {. }
$$

Then $\xi_{0}$ is regular with respect to $\Theta$.

Before the proof, let us point out some special cases. Proposition 5.4 gives us a sufficient condition for the boundary regularity of $\xi_{0} \in \partial \Theta$, and covers a wide range of situations, the most important one probably being the one already described above in Proposition 5.3. Here are some other interesting cases.

Example 5.5. In the simplest case $n=1$, every $x_{0} \in \mathbf{R}$ is regular, so one can take $E=\left\{x_{0}\right\}$. Regularity of $\xi_{0}$ is therefore guaranteed, whenever there is a curve in $\mathbf{R}^{1+1}$ approaching $\xi_{0}$ from below, i.e. with subhorizontal derivative at $\xi_{0}$; cf. Theorem 5.2.

Example 5.6. If $n=2$, then a segment in $\mathbf{R}^{2}$ with $x_{0}$ as an endpoint will do as $E$. Then $E^{\prime}$ will be a vertical two-dimensional triangle with vertex at $\xi_{0}$. More generally, if $\gamma$ is a continuous curve in $\mathbf{R}^{2}$, ending at $x_{0}$, then $B^{\prime}\left(x_{0}, r\right) \backslash \gamma$ is regular for $p$-harmonic functions at $x_{0}$ and the corresponding set $E^{\prime}$ is a two-dimensional triangle-shaped "curtain" following the curve $\gamma$. It is also possible to have disconnected $E$, i.e. $E$ consisting of suitably chosen segments accumulating at $x_{0}$.

Example 5.7. In higher dimensions, the easiest generalisation is a set $E$ which satisfies an interior cork-screw condition at $x_{0}$ (the cone considered above does). More generally, it is enough if $B^{\prime}\left(x_{0}, r\right) \backslash E$ is porous at $x_{0}$. We recall that a set $M \subset \mathbf{R}^{n}$ is porous at a point $x \in \mathbf{R}^{n}$, if there exists a constant $c \in(0,1)$ such that, for each $\varepsilon>0$, there exist $y \in \mathbf{R}^{n}$ and $r>c|x-y|$, with $|x-y|<\varepsilon$ and $M \cap B^{\prime}(y, r)=\varnothing$. See Corollary 11.25 in Björn-Björn [4] for some more general sufficient porosity conditions. A sharp condition for $E$ follows from the Wiener criterion. 
Proof of Proposition 5.4. Let $E \subset \mathbf{R}^{n}$ be closed and such that $0 \in \partial E$. Assume that $E$ is $p$-thick at 0 . Let $\Omega=B^{\prime}\left(0, r_{0}\right) \backslash E \subset \mathbf{R}^{n}$. Then 0 is regular for $p$-harmonic functions with respect to $\Omega$, by the Wiener criterion (see above).

Let $u_{j}$ be a continuous solution to the Dirichlet problem

$$
\left\{\begin{array}{l}
\Delta_{p} u_{j}(x)=\theta \min \left\{\nabla u_{j}(x) \cdot \zeta, 0\right\}-j \quad \text { in } \Omega, \\
u_{j}-f_{j} \in W_{0}^{1, p}(\Omega),
\end{array}\right.
$$

where $f_{j}(x)=j|x|, j=1,2, \ldots$. Such a function $u_{j}$ exists by Theorem 6.21 in Malý-Ziemer [36], and Corollary 6.22 in [36] implies that

$$
\lim _{x \rightarrow 0} u_{j}(x)=f_{j}(0)=0,
$$

since 0 is regular for $p$-harmonic functions. Moreover, $\Delta_{p} u_{j}<0$, i.e. $u_{j}$ is a supersolution to the $p$-harmonic equation. Since $f_{j}$ is a subsolution to the $p$-harmonic equation (by direct calculation), Lemma 3.18 in Heinonen-Kilpeläinen-Martio [18] (and the continuity of $u_{j}$ and $f_{j}$ ) shows that $u_{j} \geq f_{j}$ in $\Omega$. Let

$$
\Theta^{\prime}=\left\{(x, t):-r_{0} / \theta<t<0 \text { and } x-\eta(-t) \zeta \in \Omega\right\}
$$

and for $\xi=(x, t) \in \Theta^{\prime}$ define

$$
v_{j}(\xi)=u_{j}(x-\eta(-t) \zeta)-j t .
$$

Then

$$
\partial_{t} v_{j}(\xi)=\eta^{\prime}(-t) \nabla u_{j}(x-\eta(-t) \zeta) \cdot \zeta-j
$$

and

$$
\Delta_{p} v_{j}(\xi)=\Delta_{p} u_{j}(x-\eta(-t) \zeta)=\theta \min \left\{\nabla u_{j}(x-\eta(-t) \zeta) \cdot \zeta, 0\right\}-j<0 .
$$

Since $0 \leq \eta^{\prime} \leq \theta$, we thus have

$$
\begin{aligned}
\Delta_{p} v_{j}(\xi) & \leq \eta^{\prime}(-t) \min \left\{\nabla u_{j}(x-\eta(-t) \zeta) \cdot \zeta, 0\right\}-j \\
& \leq \eta^{\prime}(-t) \nabla u_{j}(x-\eta(-t) \zeta) \cdot \zeta-j=\partial_{t} v_{j}(\xi)
\end{aligned}
$$

i.e. $v_{j}$ is $p$-superparabolic in $\Theta^{\prime}$. As $u_{j} \geq f_{j}$ in $\Omega$, we have

$$
v_{j}(\xi) \geq j(|x-\eta(-t) \zeta|-t)=: j d(\xi) .
$$

Moreover, (5.4) and (5.5) yield

$$
\lim _{\xi \rightarrow \xi_{0}} v_{j}(\xi)=0
$$

Hence $v_{j}$ forms a strong family of barriers at $\xi_{0}=(0,0)$ with respect to $\Theta^{\prime}$, and Theorem 3.3 shows that $\xi_{0}$ is a regular boundary point with respect to $\Theta^{\prime}$.

By our assumptions, for some $0<r<\frac{1}{2} \min \left\{r_{0}, r_{0} / \theta\right\}$ we have that $\Theta_{-} \cap$ $B\left(\xi_{0}, r\right) \subset \Theta^{\prime} \cap B\left(\xi_{0}, r\right)$. Then $\xi_{0}$ is a regular boundary point with respect to $\Theta^{\prime} \cap B\left(\xi_{0}, r\right)$, and hence also with respect to $\Theta_{-}$and consequently with respect to $\Theta$, by Propositions 3.4, 3.5 and Theorem 3.11.

\section{A Petrovskiı̌ condition for $p>2$}

In this section we show that there is an increasing barrier family in the domain (6.1) at $(0,0)$, and thus the origin as final point is regular. We follow the same approach as in Lindqvist [32]: the only difference is in the choice of the function $f$ in (6.2). 
Proposition 6.1. Let $p>2$. The origin $(0,0)$ is regular with respect to the domain

$$
\begin{aligned}
\Theta=\{(x, t): & -\frac{1}{2 e}<t<0 \text { and } \\
& \left.\left(\frac{|x|}{(-t)^{1 / \lambda}}\right)^{p /(p-1)}<K(-t)^{n(p-2) / \lambda} h(t)^{\alpha(p-2)}\right\},
\end{aligned}
$$

in $\mathbf{R}^{n+1}$, where $K$ and $\alpha$ denote arbitrary positive constants, $\lambda=n(p-2)+p$ and

$$
h(t)=\frac{|\log (-t)|^{p-2}-1}{p-2} .
$$

The choice $T=-\frac{1}{2 e}$ is completely immaterial: any negative value would do, as regularity is a purely local property by Proposition 3.5 . In the interval $\left(-\frac{1}{e}, 0\right)$, the function $h$ is strictly positive, and this simplifies some of the calculations to come.

Remark 6.2. If we choose $\alpha=\frac{1}{p-2}$, then in (6.1) we obtain

$$
\left(\frac{|x|}{(-t)^{1 / \lambda}}\right)^{p /(p-1)}<K(-t)^{n(p-2) / \lambda} \frac{|\log (-t)|^{p-2}-1}{p-2},
$$

and when $p \rightarrow 2$, this formally becomes

$$
\left(\frac{|x|}{(-t)^{1 / 2}}\right)^{2}<K \log |\log (-t)|
$$

which resembles Petrovskiı̌'s condition mentioned in the introduction. Unfortunately, this result is purely formal, because when $p \rightarrow 2$, we have $M \rightarrow \infty$ and $\varepsilon \rightarrow 0$ in the proof below, and the argument becomes void. The lack of stability in our estimates is apparent also from another point of view: the constant $K$ is completely arbitrary here, whereas from Petrovskil's condition it is known that its value is very precisely determined.

Remark 6.3. In Kilpeläinen-Lindqvist [21], pp. 676-677, it is shown that the origin is an irregular boundary point with respect to the so-called Barenblatt balls; namely, it is shown that $(0,0)$ is an irregular boundary point with respect to the domain

$$
\left\{(x, t): \frac{p-2}{p \lambda^{1 /(p-1)}}\left(\frac{|x|}{(-t)^{1 / \lambda}}\right)^{p /(p-1)}<1-2^{-(p-2) /(p-1)} \text { and }-T<t<0\right\},
$$

where $T$ depends on $p$. Now, provided $t$ is small enough, which we can always assume without loss of generality, as regularity is a local property by Proposition 3.5, it is easy to check that in $\Theta$,

$$
\begin{aligned}
\frac{p-2}{p \lambda^{1 /(p-1)}}\left(\frac{|x|}{(-t)^{1 / \lambda}}\right)^{p /(p-1)} & <K \frac{p-2}{p \lambda^{1 /(p-1)}}(-t)^{n(p-2) / \lambda} h(t)^{\alpha(p-2)} \\
& <1-2^{-(p-2) /(p-1)}
\end{aligned}
$$

This suggests that there is a sort of threshold for the regularity of the final point. Once more, there is no stability in this estimate, as $p \rightarrow 2$.

Proof of Proposition 6.1. By Definition 3.2 and Theorem 3.3, it is enough to show that there exists a barrier family $\left\{w_{j}\right\}_{j=1}^{\infty}$ in $\Theta$ at the origin $\xi_{0}=(0,0)$. The family 
$\left\{w_{j}\right\}_{j=1}^{\infty}$ we construct will be smooth in $\Theta$, so that $\partial_{t} w_{j}-\Delta_{p} w_{j} \geq 0$ is satisfied in the classical sense. It will be constructed in the form

$$
\begin{aligned}
w_{j}(x, t)= & f(t)\left[j+\frac{p-2}{p \lambda^{1 /(p-1)}}\left(\frac{|x|}{(-t)^{1 / \lambda}}\right)^{p /(p-1)}\right]^{(p-1) /(p-2)} \\
& -j^{(p-1) /(p-2)} f(t)+\rho_{j}(t),
\end{aligned}
$$

where

$$
f(t)=-\varepsilon h(t)^{\alpha}=-\varepsilon\left(\frac{|\log (-t)|^{p-2}-1}{p-2}\right)^{\alpha}<0,
$$

$\varepsilon>0$ is a positive parameter to be chosen, and $\rho_{j}>0$ is a proper function to be determined. (The function $\rho_{j}$ will depend on $j$, but $\varepsilon$ will not.)

We shall select $\rho_{j}$ such that $w_{j}$ is a supersolution in the domain where $w_{j}>0$, and this domain is to contain $\Theta$. In the following, for simplicity we will drop the subscript $j$ in $w_{j}$ and $\rho_{j}$. Notice that $w$ is positive when

$$
\left[j+\frac{p-2}{p \lambda^{1 /(p-1)}}\left(\frac{|x|}{(-t)^{1 / \lambda}}\right)^{p /(p-1)}\right]^{(p-1) /(p-2)}<j^{(p-1) /(p-2)}-\frac{\rho(t)}{f(t)} .
$$

Moreover,

$$
w(x, t)<f(t) j^{(p-1) /(p-2)}-f(t) j^{(p-1) /(p-2)}+\rho(t)=\rho(t) .
$$

Thus, (b) in Definition 3.2 holds true if $\rho(t) \rightarrow 0$ as $t \rightarrow 0^{-}$. This requirement restricts the choice of $\rho$ in a decisive way. However, it turns out that $\rho$ can be chosen in such a way, that conditions (a)-(c) in Definition 3.2 can all be satisfied.

We now show that $w$ is $p$-superparabolic in the domain defined by (6.3). We will then prove that this domain contains $\Theta$, and at the same time that (c) in Definition 3.2 is satisfied on $\partial \Theta$.

We set

$$
F(x, t)=j+\frac{p-2}{p \lambda^{1 /(p-1)}}\left(\frac{|x|}{(-t)^{1 / \lambda}}\right)^{p /(p-1)} .
$$

Hence

$$
\begin{aligned}
\nabla F & =\frac{p-2}{(p-1) \lambda^{1 /(p-1)}} \frac{|x|^{(2-p) /(p-1)} x}{(-t)^{p / \lambda(p-1)}}, \\
\partial_{t} F & =\frac{p-2}{(p-1) \lambda^{p /(p-1)}}\left(\frac{|x|}{(-t)^{1 / \lambda}}\right)^{p /(p-1)} \frac{1}{-t}=\frac{p(F(x, t)-j)}{\lambda(p-1)(-t)} .
\end{aligned}
$$

Since $w(x, t)=f(t) F(x, t)^{(p-1) /(p-2)}+\varphi(t)$ with $\varphi(t)=-j^{(p-1) /(p-2)} f(t)+\rho(t)$, we have

$$
\begin{gathered}
\nabla w=f(t) \frac{F(x, t)^{1 /(p-2)}}{\lambda^{1 /(p-1)}} \frac{|x|^{(2-p) /(p-1)} x}{(-t)^{p / \lambda(p-1)}} \\
|\nabla w|^{p-2} \nabla w=|f(t)|^{p-2} f(t) \frac{F(x, t)^{(p-1) /(p-2)}}{\lambda} \frac{x}{(-t)^{p / \lambda}} .
\end{gathered}
$$

Therefore,

$$
\begin{aligned}
\Delta_{p} w= & |f(t)|^{p-2} f(t) \frac{F(x, t)^{(p-1) /(p-2)}}{\lambda} \frac{n}{(-t)^{p / \lambda}} \\
+ & |f(t)|^{p-2} f(t) \frac{F(x, t)^{1 /(p-2)}}{\lambda^{p /(p-1)}}\left(\frac{|x|}{(-t)^{1 / \lambda}}\right)^{p /(p-1)} \frac{1}{(-t)^{p / \lambda}} \\
= & |f(t)|^{p-2} f(t) \frac{F(x, t)^{(p-1) /(p-2)}}{\lambda} \frac{n}{(-t)^{p / \lambda}} \\
& +\frac{p|f(t)|^{p-2} f(t)}{\lambda(p-2)(-t)^{p / \lambda}} F(x, t)^{1 /(p-2)}(F(x, t)-j) .
\end{aligned}
$$


Moreover,

$$
\begin{aligned}
\partial_{t} w & =\varphi^{\prime}(t)+f^{\prime}(t) F(x, t)^{(p-1) /(p-2)}+\frac{p-1}{p-2} f(t) F(x, t)^{1 /(p-2)} \partial_{t} F \\
& =\varphi^{\prime}(t)+f^{\prime}(t) F(x, t)^{(p-1) /(p-2)}+\frac{p f(t) F(x, t)^{1 /(p-2)}(F(x, t)-j)}{\lambda(p-2)(-t)} .
\end{aligned}
$$

Combining the previous expressions yields

$$
\begin{aligned}
\partial_{t} w-\Delta_{p} w= & \varphi^{\prime}(t)+f^{\prime}(t) F(x, t)^{(p-1) /(p-2)}+\frac{p f(t) F(x, t)^{1 /(p-2)}(F(x, t)-j)}{\lambda(p-2)(-t)} \\
& -|f(t)|^{p-2} f(t) \frac{F(x, t)^{(p-1) /(p-2)}}{\lambda} \frac{n}{(-t)^{p / \lambda}} \\
& -\frac{p|f(t)|^{p-2} f(t)}{\lambda(p-2)(-t)^{p / \lambda}} F(x, t)^{1 /(p-2)}(F(x, t)-j) \\
= & \varphi^{\prime}(t)-\frac{p j}{\lambda(p-2)}\left(\frac{1}{-t}-\frac{|f(t)|^{p-2}}{(-t)^{p / \lambda}}\right) f(t) F(x, t)^{1 /(p-2)} \\
& +F(x, t)^{(p-1) /(p-2)}\left(f^{\prime}(t)+\frac{p f(t)}{\lambda(p-2)(-t)}-\frac{n|f(t)|^{p-2} f(t)}{\lambda(-t)^{p / \lambda}}\right. \\
& \left.-\frac{p|f(t)|^{p-2} f(t)}{\lambda(p-2)(-t)^{p / \lambda}}\right) .
\end{aligned}
$$

Since

$$
\frac{n}{\lambda}+\frac{p}{\lambda(p-2)}=\frac{1}{p-2}
$$

we obtain

$$
\begin{aligned}
\partial_{t} w-\Delta_{p} w= & \varphi^{\prime}(t)-\frac{p j}{\lambda(p-2)}\left(\frac{1}{-t}-\frac{|f(t)|^{p-2}}{(-t)^{p / \lambda}}\right) f(t) F(x, t)^{1 /(p-2)} \\
& +F(x, t)^{(p-1) /(p-2)}\left(f^{\prime}(t)+\frac{p f(t)}{\lambda(p-2)(-t)}-\frac{|f(t)|^{p-2} f(t)}{(p-2)(-t)^{p / \lambda}}\right) .
\end{aligned}
$$

We need to ensure that $\partial_{t} w-\Delta_{p} w \geq 0$. As we mentioned before, we now choose

$$
f(t)=-\varepsilon h(t)^{\alpha}=-\varepsilon\left(\frac{|\log (-t)|^{p-2}-1}{p-2}\right)^{\alpha},
$$

where $\varepsilon$ is still to be fixed. Since

$$
f^{\prime}(t)=-\alpha \varepsilon h(t)^{\alpha-1} \frac{|\log (-t)|^{p-3}}{-t}<0,
$$

we have

$$
\begin{aligned}
f^{\prime}(t) & +\frac{p f(t)}{\lambda(p-2)(-t)}-\frac{|f(t)|^{p-2} f(t)}{(p-2)(-t)^{p / \lambda}} \\
& =-\alpha \varepsilon h(t)^{\alpha-1} \frac{|\log (-t)|^{p-3}}{-t}-\frac{p \varepsilon}{\lambda(p-2)(-t)} h(t)^{\alpha}+\frac{\varepsilon^{p-1}}{(p-2)(-t)^{p / \lambda}} h(t)^{\alpha(p-1)} .
\end{aligned}
$$

This expression is certainly negative if

$$
-\frac{p \varepsilon}{\lambda(p-2)(-t)} h(t)^{\alpha}+\frac{\varepsilon^{p-1}}{(p-2)(-t)^{p / \lambda}} h(t)^{\alpha(p-1)} \leq 0,
$$

which holds if

$$
\frac{p}{\lambda} \geq g(t)^{p-2} \varepsilon^{p-2}, \quad \text { where } g(t)=(-t)^{n / \lambda} h(t)^{\alpha} .
$$


Letting $M=\sup _{-1 / 2 e<t<0} g(t)$, which is finite and positive, the negativity condition becomes

$$
\frac{p}{\lambda}=\varepsilon^{p-2} M^{p-2} \text {. }
$$

We fix $\varepsilon$ in this way; note that $\varepsilon$ depends on $n, p$ and $\alpha$. Once $\varepsilon$ is chosen, it is easy to check that

$$
-\frac{p j}{\lambda(p-2)}\left(\frac{1}{-t}-\frac{|f(t)|^{p-2}}{(-t)^{p / \lambda}}\right) f(t)>0,
$$

as $p / \lambda<1$. Relying on (6.4) and (6.5), we have in the domain defined by (6.3),

$$
\begin{aligned}
\partial_{t} w-\Delta_{p} w \geq & \varphi^{\prime}(t)-\frac{p j}{\lambda(p-2)}\left(\frac{1}{-t}-\frac{|f(t)|^{p-2}}{(-t)^{p / \lambda}}\right) f(t) j^{1 /(p-2)} \\
& +\left(f^{\prime}(t)+\frac{p f(t)}{\lambda(p-2)(-t)}-\frac{|f(t)|^{p-2} f(t)}{(p-2)(-t)^{p / \lambda}}\right)\left(j^{(p-1) /(p-2)}-\frac{\rho(t)}{f(t)}\right) \\
= & \rho^{\prime}(t)+\frac{n \varepsilon^{p-1} j^{(p-1) /(p-2)}}{\lambda(-t)^{p / \lambda}} h(t)^{\alpha(p-1)} \\
& +\rho(t)\left(\frac{-\alpha|\log (-t)|^{p-3}}{-t h(t)}-\frac{p}{\lambda(p-2)(-t)}+\frac{\varepsilon^{p-2} h(t)^{\alpha(p-2)}}{(p-2)(-t)^{p / \lambda}}\right),
\end{aligned}
$$

where we have taken into account that $\varphi(t)=\rho(t)-j^{(p-1) /(p-2)} f(t)$. Now we choose $\rho$ in such a way that the expression on the right-hand side is positive. We let

$$
\rho(t)=A(-t)^{1-p / \lambda} h(t)^{\alpha(p-1)}=A(-t)^{1-p / \lambda}\left(\frac{|\log (-t)|^{p-2}-1}{p-2}\right)^{\alpha(p-1)},
$$

where $A>0$ is to be determined. As $p<\lambda, \lim _{t \rightarrow 0^{-}} \rho(t)=0$ as required. Since

$$
\begin{aligned}
\rho^{\prime}(t)= & -A \frac{n(p-2)}{\lambda}(-t)^{-p / \lambda} h(t)^{\alpha(p-1)} \\
& +A \alpha(p-1)(-t)^{1-p / \lambda} h(t)^{\alpha(p-1)-1} \frac{|\log (-t)|^{p-3}}{-t},
\end{aligned}
$$

we obtain

$$
\begin{aligned}
\partial_{t} w-\Delta_{p} w \geq & \frac{1}{\lambda(-t)^{p / \lambda}}\left(-A n(p-2)+n \varepsilon^{p-1} j^{(p-1) /(p-2)}-\frac{A p}{(p-2)}\right) h(t)^{\alpha(p-1)} \\
& +\frac{A \alpha(p-2)}{(-t)^{p / \lambda}} h(t)^{\alpha(p-1)-1}|\log (-t)|^{p-3}+\frac{A \varepsilon^{p-2}(-t)^{1-2 p / \lambda}}{p-2} h(t)^{\alpha(2 p-3)} .
\end{aligned}
$$

We conclude that $\partial_{t} w-\Delta_{p} w \geq 0$, provided we choose $A>0$ such that

$$
-A\left(n(p-2)+\frac{p}{p-2}\right)+n \varepsilon^{p-1} j^{(p-1) /(p-2)} \geq 0,
$$

which holds when we let

$$
A=\frac{n(p-2) \varepsilon^{p-1} j^{(p-1) /(p-2)}}{n(p-2)^{2}+p} .
$$

Relying on the choice of $\varepsilon, A, f$ and $\rho$ in (6.6)-(6.9), we can now rewrite (6.3) in the following way

$$
\begin{aligned}
& {\left[1+\frac{p-2}{p j \lambda^{1 /(p-1)}}\left(\frac{|x|}{(-t)^{1 / \lambda}}\right)^{p /(p-1)}\right]^{(p-1) /(p-2)}} \\
& <1+\frac{n p(p-2)}{\left[n(p-2)^{2}+p\right] \lambda M^{p-2}}(-t)^{n(p-2) / \lambda} h(t)^{\alpha(p-2)}
\end{aligned}
$$


Notice that $j$ is still completely undetermined. In order to prove (a) in Definition 3.2, we need to show that the domain defined by (6.10) contains $\Theta$. Indeed, by (6.1), we have

$$
\left(\frac{|x|}{(-t)^{1 / \lambda}}\right)^{p /(p-1)}<K M^{p-2} \text { in } \Theta .
$$

Taking into account that $(1+s)^{(p-1) /(p-2)} \leq 1+\frac{p-1}{p-2} s(1+s)^{1 /(p-2)}$ for $s \geq 0$ yields

$$
\begin{aligned}
& {\left[1+\frac{p-2}{p j \lambda^{1 /(p-1)}}\left(\frac{|x|}{(-t)^{1 / \lambda}}\right)^{p /(p-1)}\right]^{(p-1) /(p-2)}} \\
& \quad \leq 1+\frac{p-1}{p j \lambda^{1 /(p-1)}}\left(\frac{|x|}{(-t)^{1 / \lambda}}\right)^{p /(p-1)}\left(1+\frac{p-2}{p j \lambda^{1 /(p-1)}} K M^{p-2}\right)^{1 /(p-2)} \\
& \quad \leq 1+\frac{L}{j}\left(\frac{|x|}{(-t)^{1 / \lambda}}\right)^{p /(p-1)} \\
& \quad<1+\frac{L}{j} K(-t)^{n(p-2) / \lambda} h(t)^{\alpha(p-2)}
\end{aligned}
$$

where $L$ is independent of $j \geq 1$. Since both $K$ and $M$ are independent of $j$, provided we choose $j$ large enough, we can always conclude that

$$
\frac{L}{j} K<\frac{n p(p-2)}{\left[n(p-2)^{2}+p\right] \lambda M^{p-2}},
$$

therefore satisfying (6.10).

Finally, we show that (c) in Definition 3.2 is satisfied. Let $y(t),-1 / 2 e<t<0$, be the solution to

$$
\left(\frac{y}{(-t)^{1 / \lambda}}\right)^{p /(p-1)}=K(-t)^{n(p-2) / \lambda} h(t)^{\alpha(p-2)} .
$$

Then for $(x, t) \in \partial \Theta$ with $|x|=y(t)$, we have

$$
\begin{aligned}
w(x, t)= & -\varepsilon h(t)^{\alpha}\left(j+\frac{p-2}{p \lambda^{1 /(p-1)}} K(-t)^{n(p-2) / \lambda} h(t)^{\alpha(p-2)}\right)^{(p-1) /(p-2)} \\
& +\varepsilon j^{(p-1) /(p-2)} h(t)^{\alpha}+A(-t)^{n(p-2) / \lambda} h(t)^{\alpha(p-1)} \\
= & -\varepsilon j^{(p-1) /(p-2)} h(t)^{\alpha}\left(1+\frac{K(p-2)}{p j \lambda^{1 /(p-1)}}(-t)^{n(p-2) / \lambda} h(t)^{\alpha(p-2)}\right)^{(p-1) /(p-2)} \\
& +\varepsilon j^{(p-1) /(p-2)} h(t)^{\alpha}+A(-t)^{n(p-2) / \lambda} h(t)^{\alpha(p-1)} .
\end{aligned}
$$

Using that $(1+s)^{(p-1) /(p-2)} \leq 1+B s$ for $0 \leq s \leq \gamma$ and that $B$ only depends on the bound $\gamma$, we have with $\widetilde{K}=K B$,

$$
\begin{aligned}
w(x, t) \geq & -\varepsilon j^{(p-1) /(p-2)} h(t)^{\alpha}\left(1+\frac{\widetilde{K}(p-2)}{p j \lambda^{1 /(p-1)}}(-t)^{n(p-2) / \lambda} h(t)^{\alpha(p-2)}\right) \\
& +\varepsilon j^{(p-1) /(p-2)} h(t)^{\alpha}+A(-t)^{n(p-2) / \lambda} h(t)^{\alpha(p-1)} \\
= & -\varepsilon j^{(p-1) /(p-2)} \frac{\widetilde{K}(p-2)}{p j \lambda^{1 /(p-1)}}(-t)^{n(p-2) / \lambda} h(t)^{\alpha(p-1)} \\
& +A(-t)^{n(p-2) / \lambda} h(t)^{\alpha(p-1)} \\
= & \varepsilon j^{(p-1) /(p-2)}\left(\frac{n(p-2) \varepsilon^{p-2}}{n(p-2)^{2}+p}-\frac{\widetilde{K}(p-2)}{p j \lambda^{1 /(p-1)}}\right)(-t)^{n(p-2) / \lambda} h(t)^{\alpha(p-1)} .
\end{aligned}
$$


For $(x, t) \in \partial \Theta$ with $t=-\frac{1}{2 e}$ and $|x|<y(t)$, we instead have

$$
w(x, t) \geq w\left(x^{\prime}, t\right)
$$

where $\left|x^{\prime}\right|=y(t)$, since $f$ is a negative function. Together with the last estimate, this shows that $\left\{w_{j}\right\}_{j=k}^{\infty}$ is a barrier family, provided $k$ is large enough.

\section{Remarks on the regularity of a final point for $1<p<2$}

Due to the analogies in the definition of the Barenblatt fundamental solution for $p>2$ and $\frac{2 n}{n+1}<p<2$, one would expect that in the singular supercritical range $\frac{2 n}{n+1}<p<2$ the origin $(0,0)$ is regular with respect to the domain

$$
\begin{aligned}
\Theta= & \left\{(x, t) \in \mathbf{R}^{n+1}:-\frac{1}{2 e}<t<0\right. \text { and } \\
& \left.\left(\frac{|x|}{(-t)^{1 / \lambda}}\right)^{p /(p-1)}<K(-t)^{n(2-p) / \lambda}\left(\frac{|\log (-t)|^{2-p}-1}{2-p}\right)^{\alpha(2-p)}\right\},
\end{aligned}
$$

where $K$ and $\alpha$ denote arbitrary positive constants, and $\lambda=n(p-2)+p$. Notice that the above-indicated range of $p$ ensures that $\lambda>0$.

With methods that are very similar to the ones used in the previous section, it is not hard to prove that for proper values of $\varepsilon$ and for sufficiently large values of $j$, the function

$$
\begin{aligned}
w= & -\varepsilon\left(\frac{|\log (-t)|^{2-p}-1}{2-p}\right)^{\alpha}\left[j-\frac{2-p}{p \lambda^{1 /(p-1)}}\left(\frac{|x|}{(-t)^{1 / \lambda}}\right)^{p /(p-1)}\right]^{-(p-1) /(2-p)} \\
& +\varepsilon j^{-(p-1) /(2-p)}\left(\frac{|\log (-t)|^{2-p}-1}{2-p}\right)^{\alpha} \\
& +\frac{n(2-p) \varepsilon j^{-(p-1) /(2-p)}}{\lambda M^{2-p}}(-t)^{n(2-p) / \lambda}\left(\frac{|\log (-t)|^{2-p}-1}{2-p}\right)^{\alpha(3-p)}
\end{aligned}
$$

is one barrier. Unfortunately, unlike the $p>2$ case, now as $j \rightarrow \infty, w \rightarrow 0$ and condition (c) of Definition 3.2 cannot be satisfied. Therefore, we do not have a whole family of barriers, and we cannot conclude regularity.

What we can prove is a somewhat weaker result, valid in the whole singular range $1<p<2$.

Proposition 7.1. Let $1<p<2$. The origin $(0,0)$ is regular with respect to the domain

$$
\Theta=\left\{(x, t) \in \mathbf{R}^{n+1}:-1<t<0 \text { and }|x|^{l}<K(-t)\right\}
$$

if $0<l<p$ and $K>1$ is arbitrary.

Besides being nonoptimal, the result of Proposition 7.1 is also not stable, and it cannot be: Indeed, the barrier $u_{j}$ below is defined only for $1<p<2$ and for $p=2$ the regularity of the origin as an end point is characterised by the original Petrovskiı criterion.

Proof. Let

$$
G^{j}=\left\{x:|x|<\frac{1}{2} \sqrt{\frac{2-p}{j}}\right\} \times(-\infty, 0),
$$


and consider the function $u_{j}: G^{j} \rightarrow \mathbf{R}$ defined by

$$
u_{j}(x, t)=j^{\alpha}\left(-\frac{t}{j}\right)^{1 /(2-p)}\left(\frac{2-p}{j}-|x|^{2}\right),
$$

where $\alpha$ is a positive parameter to be determined and $j>1$ is arbitrary. First of all, notice that

$$
\lim _{G^{j} \ni(x, t) \rightarrow(0,0)} u_{j}(x, t)=0 .
$$

Next, we want to show that $u_{j}$ is $p$-superparabolic in $G^{j}$, provided $\alpha$ is properly chosen. We have

$$
\begin{aligned}
\partial_{t} u_{j} & =-\frac{j^{\alpha-1}}{2-p}\left(-\frac{t}{j}\right)^{(p-1) /(2-p)}\left(\frac{2-p}{j}-|x|^{2}\right), \\
\Delta_{p} u_{j} & =-2^{p-1} j^{\alpha(p-1)}\left(-\frac{t}{j}\right)^{(p-1) /(2-p)}(n+p-2)|x|^{p-2} .
\end{aligned}
$$

Therefore in $G^{j}$,

$$
\begin{aligned}
\partial_{t} u_{j} & -\Delta_{p} u_{j} \\
& =\left(-\frac{t}{j}\right)^{(p-1) /(2-p)}\left[2^{p-1} j^{\alpha(p-1)}(n+p-2)|x|^{p-2}-\frac{j^{\alpha-1}}{2-p}\left(\frac{2-p}{j}-|x|^{2}\right)\right] \\
& \geq\left(-\frac{t}{j}\right)^{(p-1) /(2-p)}\left[2^{p-1} j^{\alpha(p-1)}(n-1)\left(\frac{1}{|x|}\right)^{2-p}-j^{\alpha-2}\right] \\
& \geq\left(-\frac{t}{j}\right)^{(p-1) /(2-p)}\left[\frac{2}{(2-p)^{1-p / 2}}(n-1) j^{\alpha(p-1)+1-p / 2}-j^{\alpha-2}\right] \\
& \geq\left(-\frac{t}{j}\right)^{(p-1) /(2-p)}\left(j^{\alpha(p-1)+1-p / 2}-j^{\alpha-2}\right),
\end{aligned}
$$

and, since $j>1$, we have shown that $u_{j}$ is $p$-superparabolic if $\alpha>0$ is such that

$$
\alpha(p-1)+\frac{2-p}{2}>\alpha-2, \quad \text { or equivalently } \quad \alpha<\frac{2}{2-p}+\frac{1}{2} .
$$

Finally, we show that condition (c) in Definition 3.2 is satisfied, provided $\alpha$ is properly chosen. Let

$$
m_{j}=\inf _{(x, t) \in \Theta \cap \partial G^{j}} u_{j}(x, t)>0 .
$$

Since for any $(x, t) \in G^{j}$ we have

$$
u_{j}(x, t) \geq \frac{3(2-p)}{4} j^{\alpha-1}\left(-\frac{t}{j}\right)^{1 /(2-p)}=\frac{3(2-p)}{4} j^{\alpha-1-1 /(2-p)}(-t)^{1 /(2-p)},
$$

we see that $m_{j}=u_{j}(x, t)$ if $|x|=\frac{1}{2} \sqrt{(2-p) / j}$ and $t=-|x|^{l} / K$, i.e.

$$
m_{j}=\frac{3(2-p)}{4}\left(\frac{\left(\frac{1}{2} \sqrt{2-p}\right)^{l}}{K}\right)^{1 /(2-p)} j^{\alpha-1-(1+l / 2) /(2-p)} .
$$

Since also $u_{j}(x, t) \geq m_{j}$ when $(x, t) \in \partial\left(\Theta \cap G^{j}\right)$ and $t=-1$, it follows from the pasting lemma (Lemma 2.9) that

$$
w_{j}= \begin{cases}\min \left\{u_{j}, m_{j}\right\} & \text { in } G^{j} \cap \Theta, \\ m_{j} & \text { in } \Theta \backslash G^{j}\end{cases}
$$


is a barrier family, provided we choose $\alpha$ so that

$$
1+\frac{1+\frac{1}{2} l}{2-p}<\alpha<\frac{2}{2-p}+\frac{1}{2},
$$

which is possible as $0<l<p$.

\section{Open problems}

Here we collect a short list of open problems.

1. In Theorem 3.3 we characterise the regularity of a boundary point in terms of the existence of a family of barriers. Is it really necessary to have a family of barriers? Or, stated otherwise, does there exist an open set $\Theta$ and an irregular boundary point $\xi_{0} \in \partial \Theta$ such that there is one barrier at $\xi_{0}$ ? We think that this question has a positive answer, but unfortunately, we could not find such an example.

2. The previous question is directly linked to the stability as $p \rightarrow 2$ of the results given in Sections 6 and 7: if a family is indeed necessary, then, in order to prove stability, one should be able to find a family of barriers, which in the limit for $p=2$ converges to a set of barriers, all multiples of a minimal one; this has to be the case, because a single barrier suffices when $p=2$.

3. When $p>2$, can we stabilise the estimates of Proposition 6.1 and recover the classical Petrovskiŭ condition?

4. In the range $1<p<2$ (or at least $\frac{2 n}{n+2}<p<2$ ) can we improve the result of Proposition 7.1 and build a family of barriers, which in the limit as $p \rightarrow 2^{-}$ yields the original Petrovskil condition?

5. The problem of the north pole for the exterior ball condition remains open, when $n>1$.

6. As we mentioned in Section 5.2, it would be interesting to see, whether the so-called tusk condition holds for a general $p>1$, and not just for $p=2$.

7. A related interesting problem is the resolutivity problem: If $f \in C(\Theta)$ is then always $\bar{H} f=\underline{H} f$ ?

\section{References}

1. T. Adamowicz, A. BJÖRn and J. BJöRn, Regularity of $p(\cdot)$-superharmonic functions, the Kellogg property and semiregular boundary points, to appear in Ann. Inst. H. Poincaré Anal. Non Lineaire.

2. G. I. Barenblatt, On some unsteady motions of a liquid or a gas in a porous medium, Prikl. Mat. Mech. 16 (1952), 67-78.

3. A. BJÖRN and J. BJÖRN, Boundary regularity for $p$-harmonic functions and solutions of the obstacle problem, J. Math. Soc. Japan 58 (2006), 1211-1232.

4. A. BJörn and J. BJÖRn, Nonlinear Potential Theory on Metric Spaces, EMS Tracts in Mathematics 17, European Math. Soc., Zurich, 2011.

5. J. BJÖRN, Wiener criterion for Cheeger $p$-harmonic functions on metric spaces, in Potential Theory in Matsue, Advanced Studies in Pure Mathematics 44, pp. 103-115, Mathematical Society of Japan, Tokyo, 2006.

6. J. BJöRN, Fine continuity on metric spaces, Manuscripta Math. 125 (2008), 369-381.

7. J. Buörn, P. MacManus and N. Shanmugalingam, Fat sets and pointwise boundary estimates for $p$-harmonic functions in metric spaces, J. Anal. Math. 85 (2001), 339-369. 
8. J. Bliedtner, and W. Hansen, Potential Theory, Universitext, Springer, Berlin, 1986.

9. E. DiBenedetto, Degenerate Parabolic Equations, Universitext, Springer, New York, 1993.

10. E. DiBenedetto, U. Gianazza and V. Vespri, Harnack's Inequality for Degenerate and Singular Parabolic Equations, Springer Monographs in Mathematics, Springer, New York, 2012.

11. J. L. Doob, Classical Potential Theory and its Probabilistic Counterpart, Grundlehren Math. Wiss. 262, Springer, New York, 1984.

12. E. EFfros and J. L. KAZDAN, On the Dirichlet problem for the heat equation, Indiana Univ. Math. J. 20 (1970), 683-693.

13. L. C. Evans and R. F. Gariepy, Wiener's criterion for the heat equation, Arch. Ration. Mech. Anal. 78 (1982), 293-314.

14. M. Fontes, Initial-boundary value problems for parabolic equations, Ann. Acad. Sci. Fenn. Math. 34 (2009), 583-605.

15. V. A. Galaktionov, On regularity of a boundary point for higher-order parabolic equations: towards Petrovskii-type criterion by blow-up approach, NoDEA Nonlinear Differential Equations Appl. 16 (2009), 597-655.

16. R. GAriepy and W. P. Ziemer, Behavior at the boundary of solutions of quasilinear elliptic equations, Arch. Rational Mech. Anal. 56 (1974/75), $372-384$

17. S. Granlund, P. Lindqvist and O. Martio, Note on the PWB method in the non-linear case, Pacific J. Math. 125 (1986), 381-395.

18. J. Heinonen, T. Kilpeläinen and O. Martio, Nonlinear Potential Theory of Degenerate Elliptic Equations, 2nd ed., Dover, Mineola, NY, 2006.

19. P.-A. Ivert, On the boundary value problem for $p$-parabolic equations, in Methods of Spectral Analysis in Mathematical Physics, Oper. Theory Adv. Appl. 186, pp. 229-239, Birkhäuser, Basel, 2009.

20. P. JuUtinen and P. Lindqvist and J.J. Manfredi, On the equivalence of viscosity solutions and weak solutions for a quasi-linear equation, SIAM J. Math. Anal. 33 (2001), 699-717.

21. T. Kilpeläinen and P. Lindqvist, On the Dirichlet boundary value problem for a degenerate parabolic equation, SIAM J. Math. Anal. 27 (1996), 661-683.

22. T. KilpeläInen and J. MALÝ, The Wiener test and potential estimates for quasilinear elliptic equations, Acta Math. 172 (1994), 137-161.

23. J. Kinnunen and P. Lindqvist, Summability of semicontinuous supersolutions to a quasilinear parabolic equation, Ann. Sc. Norm. Super. Pisa Cl. Sci. 4 (2005), 59-78.

24. J. Kinnunen and P. Lindqvist, Pointwise behaviour of semicontinuous supersolutions to a quasilinear parabolic equation, Ann. Mat. Pura Appl. 185 (2006), 411-435.

25. R. Korte, T. Kuusi and M. Parviainen, A connection between a general class of superparabolic functions and supersolutions, J. Evol. Equ. 10 (2010), $1-20$.

26. R. Korte, T. Kuusi and J. Siljander, Obstacle problem for nonlinear parabolic equations, J. Differential Equations 246 (2009), 3668-3680.

27. T. KuUsi, Harnack estimates for weak supersolutions to nonlinear degenerate parabolic equations, Ann. Sc. Norm. Super. Pisa Cl. Sci. 7 (2008), 673-716.

28. T. KuUsi, Lower semicontinuity of weak supersolutions to nonlinear parabolic equations, Differential Integral Equations 22 (2009), 1211-1222.

29. H. Lebesgue, Sur des cas d'impossibilité du problème de Dirichlet ordinaire, in Vie de la société (in the part C. R. Séances Soc. Math. France (1912)), p. 17, Bull. Soc. Math. France 41 (1913), 1-62 (supplément éspecial). 
30. H. Lebesgue, Conditions de régularité, conditions d'irrégularité, conditions d'impossibilité dans le problème de Dirichlet, C. R. Acad. Sci. Paris 178 (1924), 349-354.

31. G. M. Lieberman, Intermediate Schauder theory for second order parabolic equations. III. The tusk conditions, Appl. Anal. 33 (1989), 25-43.

32. P. Lindqvist, A criterion of Petrowsky's kind for a degenerate quasilinear parabolic equation, Revista Mat. Iberoam. 11 (1995), 569-578.

33. P. Lindqvist, Regularity of supersolutions, in Regularity Estimates for Nonlinear Elliptic and Parabolic Problems, Lecture Notes in Math. 2045, pp. 73-131, Springer, Berlin-Heidelberg, 2012.

34. P. Lindqvist and O. Martio, Two theorems of N. Wiener for solutions of quasilinear elliptic equations, Acta Math. 155 (1985), 153-171.

35. P. Lindqvist and M. Parviainen, Irregular time dependent obstacles, J. Funct. Anal. 263 (2012), 2458-2482.

36. J. Malý and W. P. Ziemer, Fine Regularity of Solutions of Elliptic Partial Differential Equations, Math. Surveys and Monographs 51, Amer. Math. Soc., Providence, RI, 1997.

37. V. G. MAZ'YA, On the continuity at a boundary point of solutions of quasilinear elliptic equations, Vestnik Leningrad. Univ. Mat. Mekh. Astronom. 25:13 (1970), 42-55 (Russian). English transl.: Vestnik Leningrad Univ. Math. 3 (1976), 225-242.

38. P. Mikkonen, On the Wolff Potential and Quasilinear Elliptic Equations Involving Measures, Ann. Acad. Sci. Fenn. Math. Dissertationes 104 (1996).

39. O. Perron, Eine neue Behandlung der ersten Randwertaufgabe für $\Delta u=0$, Math. Z. 18 (1923), 42-54.

40. I. Petrovskĭ, Über die Lösungen der ersten Randwertaufgabe der Wärmeleitungsgleichung, Uchebn. Zapiski Moskov. Gos. Univ. 2 (1934), 55-59.

41. I. PetrovskIĬ, Zur ersten Randwertaufgabe der Wärmeleitungsgleichung, Compos. Math. 1 (1935), 383-419.

42. N. Wiener, The Dirichlet problem, J. Math. Phys. 3 (1924), 127-146.

43. N. A. Watson, Introduction to Heat Potential Theory, Math. Surveys and Monographs 182, Amer. Math. Soc., Providence, RI, 2012. 